\title{
STABILITY OF SYMMETRIC POWERS OF VECTOR BUNDLES OF RANK TWO WITH EVEN DEGREE ON A CURVE
}

\author{
JEONG-SEOP KIM
}

\begin{abstract}
This paper treats the strict semi-stability of the symmetric powers $S^{k} E$ of a stable vector bundle $E$ of rank 2 with even degree on a smooth projective curve $C$ of genus $g \geq 2$. The strict semistability of $S^{2} E$ is equivalent to the orthogonality of $E$ or the existence of a bisection on the ruled surface $\mathbb{P}_{C}(E)$ whose self-intersection number is zero. A relation between the two interpretations is investigated in this paper through elementary transformations. This paper also gives a classification of $E$ with strictly semi-stable $S^{3} E$. Moreover, it is shown that when $S^{2} E$ is stable, every symmetric power $S^{k} E$ is stable for all but a finite number of $E$ in the moduli of stable vector bundles of rank 2 with fixed determinant of even degree on $C$.
\end{abstract}

\section{INTRODUCTION}

All varieties are defined over the field of complex numbers $\mathbb{C}$. Let $C$ be a smooth projective curve and $E$ be a vector bundle on $C$ of $\operatorname{rank} r=\operatorname{rk} E$ and degree $d=\operatorname{deg} E$. Then $E$ is said to be stable (resp. semi-stable) if, for every nonzero proper subbundle $F$ of $E$ with torsion-free quotient $E / F$, the slope $\mu(F)$ of $F$ is less than (resp. less than or equal to) $\mu(E)$ where the slope is defined by $\mu(F)=\operatorname{deg} F / \operatorname{rk} F$ [9. p. 87]. We may assume that the quotients are locally free since a torsion-free sheaf is locally free on $C$. A semi-stable vector bundle $E$ is called strictly semi-stable if $E$ is not stable.

It is natural to investigate the stability of the symmetric power $S^{k} E$ of $E$ when $E$ is stable. Using the correspondence between the stability of $E$ and the irreducibility of its associated unitary representation $\rho_{E}: \pi_{1}(C) \rightarrow U(r)$ identified by Narasimhan and Seshadri [17, it is possible to prove the following.

Theorem ([11, p. 53]). Let $C$ be a smooth projective curve of genus $g \geq 2$ and $E$ be a stable vector bundle on $C$. Then

(1) $S^{k} E$ is semi-stable for every $k \geq 2$ for all $E$, and

(2) $S^{k} E$ is stable for every $k \geq 2$ for sufficiently general $E$.

The next question would be a classification of stable $E$ for which $S^{k} E$ is strictly semi-stable. Because we are interested in the case where $r=2$ and $d$ is even, we may assume that $\operatorname{det} E=\mathcal{O}_{C}$ after substituting $E$ by $E \otimes L^{-1}$ for some line bundle $L$ with $L^{2}=\operatorname{det} E$. Then $E$ is self-dual as $E \cong E^{\vee} \otimes(\operatorname{det} E) \cong E^{\vee}$. Note that $S^{k}\left(E \otimes L^{-1}\right)=S^{k} E \otimes L^{-k}$ and the stability is invariant under the twist by a line bundle. As

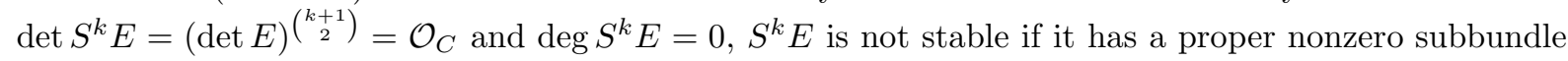
$V \rightarrow S^{k} E$ of $\operatorname{deg} V=0$ with the quotient vector bundle $Q$ of $\operatorname{deg} Q=0$. By taking the dual of the quotient $S^{k} E \rightarrow Q$ together with the self-duality of $S^{k} E$ given by

$$
\left(S^{k} E\right)^{\vee} \cong S^{k} E^{\vee} \cong S^{k} E
$$

we get a subbundle $Q^{\vee} \rightarrow S^{k} E$ of $\operatorname{deg} Q^{\vee}=-\operatorname{deg} Q=0$ and $\operatorname{rk} V+\operatorname{rk} Q^{\vee}=\operatorname{rk} V+\operatorname{rk} Q=\operatorname{rk} S^{k} E=k+1$. Thus $S^{k} E$ has destabilizing subbundles $V$ and $Q^{\vee}$, one of which has rank less than or equal to $\frac{k+1}{2}$ (see also [6, Proposition 2.1]). 
Remark 1.1. Let $E$ be a stable vector bundle on $C$ of rank 2 with even degree. Then $S^{k} E$ is strictly semi-stable if and only if it is destabilized by a subbundle $V \rightarrow S^{k} E$ of $\operatorname{rk} V \leq \frac{k+1}{2}$. Similarly, it suffices to consider the quotient bundles $S^{k} E \rightarrow Q$ of $\operatorname{rk} Q \leq \frac{k+1}{2}$ to determine the stability of $S^{k} E$.

By the remark and assuming the stability of lower symmetric powers, we have the following reduction.

Theorem 4.2, Let $C$ be a smooth projective curve of genus $g \geq 2$ and $E$ be a stable vector bundle on $C$ of rank 2 with even degree. Then $S^{k} E$ is stable for all $k \geq 2$ unless one of the following cases occurs.

(1) $S^{2} E$ is destabilized by a vector bundle of rank 1

(2) $S^{3} E$ is destabilized by a vector bundle of rank 2

(3) $S^{4} E$ is destabilized by a vector bundle of rank 2

(4) $S^{6} E$ is destabilized by a vector bundle of rank 3

In particular, $S^{k} E$ is stable for every $k \geq 2$ if $S^{m} E$ is stable for all $m \leq 6$.

We will classify cases (1), (2) in Section 2, 3, and give a proof for the following result in Section 4.

Theorem 4.8. If $S^{2} E$ is stable, then $S^{k} E$ is stable for every $k \geq 3$ except for finitely many $E$ in the moduli of stable vector bundles on $C$ of rank 2 with fixed determinant of even degree.

For $k=2, S^{2} E$ is strictly semi-stable if and only if it is destabilized by a quotient line bundle $S^{2} E \rightarrow L$ of $\operatorname{deg} L=0$. We will observe that this is equivalent to saying that $E$ is orthogonal where an orthogonal bundle is defined by a vector bundle $E$ with a nondegenerate symmetric bilinear form $E \otimes E \rightarrow L$ for some line bundle $L$. According to Mumford's classification [16, the orthogonal bundles of rank 2 are given by the direct images of line bundles on an unramified double covering $B \rightarrow C$. We will see that they form a positive dimensional family with fixed determinant.

On the other hand, Choi and Park [8] show that there exists a stable $E$ whose associated ruled surface $\mathbb{P}_{C}(E)$ admits a bisection of zero self-intersection using elementary transformations. Then its symmetric square $S^{2} E$ is strictly semi-stable due to the following correspondence.

$$
\left\{k \text {-sections } D \text { on } \mathbb{P}_{C}(E) \text { with } D^{2}=2 k b\right\} \leftrightarrow\left\{\text { line subbundles } L^{-1} \rightarrow S^{k} E \text { with } \operatorname{deg} L=b\right\}
$$

In Section 2, we will see a relation between orthogonal bundles and such ruled surfaces, and find that every orthogonal bundle can be obtained by the method of Choi and Park [8].

For $k=3$, since $S^{3} E$ is of rank 4 , if not stable, it is destabilized by a subbundle of rank 1 or 2 .

Theorem 3.3 and 3.11, Let $C$ be a smooth projective curve of genus $g \geq 2$ and $E$ be a stable vector bundle on $C$ of rank 2 with trivial determinant. Then

(1) $S^{3} E$ is destabilized by a subbundle of rank 1 only if $S^{2} E$ is strictly semi-stable, and there exist only a finite number of such $E$,

(2) $S^{3} E$ is destabilized by a subbundle of rank 2 if $S^{2} E$ is strictly semi-stable, and there are only finitely many such $E$ with stable $S^{2} E$.

In particular, except a finite number of $E, S^{3} E$ is strictly semi-stable if and only if $S^{2} E$ is not stable.

In Section 3, we will classify the exceptional cases as the ones satisfying $S^{2} E=\eta_{*} R$ for some unramified cyclic 3-covering $\eta: C^{\prime} \rightarrow C$ and $R \in J^{0}\left(C^{\prime}\right) \backslash \eta^{*} J^{0}(C)$ with $R^{2}=\mathcal{O}_{C^{\prime}}$. It also completes the description of $E$ with $S^{4} E$ being destabilized by a line subbundle (see Proposition 3.1). 
For $k \geq 4$, the remaining cases for the stability of $S^{k} E$ are $k=4$ and 6 as stated in Theorem 4.2. In Section 4 , we will show that each case is further reduced to the case where $S^{l} E$ is destabilized by a line subbundle for some $l \geq k$. Then, with the aid of the following corollary, we obtain the result that there are only finitely many $E$ with trivial determinant where $S^{2} E$ is stable but $S^{k} E$ is not stable for some $k \geq 3$.

Corollary 4.5. Let $C$ be a smooth projective curve of genus $g \geq 2$ and $E$ be a stable vector bundle on $C$ of rank 2 with trivial determinant. If $k \geq 3$, then there exist at most finitely many $E \in \mathcal{S U}_{C}\left(2, \mathcal{O}_{C}\right)$ where $S^{k} E$ is destabilized by a line subbundle but $S^{m} E$ is not destabilized by a line subbundle for any $m<k$.

Under the correspondence between the line subbundles of $S^{k} E$ and the $k$-sections on $X=\mathbb{P}_{C}(E)$, the result says that if $S^{2} E$ is stable and $E$ has even degree, then there are only a finite number of $X$ which admits a $k$-section of zero self-intersection for some $k \geq 3$. Notice that the class of $k$-secant divisors of zero self-intersection lies on the boundary of the closure of the cone of curves $\overline{\mathrm{NE}}(X)$ in $N_{1}(X)$ when $E$ is stable [14, I: p. 70]. Thus the result also answers to the question of how many $X=\mathbb{P}_{C}(E)$ has closed $\mathrm{NE}(X)$ for the stable vector bundles $E$ of rank 2 with even degree when $S^{2} E$ is stable.

Due to an observation by Rosoff [21, p. 123], when $D$ is the $k$-section on $X=\mathbb{P}_{C}(E)$ corresponding to a destabilizing line subbundle of $S^{k} E$, the induced $k$-covering $\pi: D \rightarrow C$ is necessarily unramified. Also, the covering gives a destabilizing quotient line bundle $\pi^{*} E \rightarrow R$ so that $\pi^{*} E$ is not stable on $D$. Further, we can derive a stronger assertion that $\pi^{*} E$ is not only strictly semi-stable but it also splits into the direct sum of line bundles, and the line bundles are torsion elements in the Picard group when $k \geq 3$. By composing a cyclic covering over which trivializes the torsion line bundles, we can conclude that $E$ is trivialized over an unramified finite covering of $C$. Namely, $E$ satisfies the property called étale triviality [4, which is known to be equivalent to saying that $E$ is a finite bundle introduced by Nori [18.

Remark 4.14. Let $E$ be a vector bundle on $C$ of rank 2 with trivial determinant. Assume that $S^{2} E$ is stable. Then $E$ is finite if and only if $S^{k} E$ is not stable for some $k \geq 3$.

Acknowledgement. I would like to thank my thesis advisor, Prof. Yongnam Lee, for introducing this topic and giving valuable guidance. I am also indebted to an anonymous reviewer of an earlier manuscript not only for providing helpful suggestions to improve the presentation but also for pointing out a flaw in the previous proof of Theorem 4.2, This work will be part of my Ph.D. thesis. I was partly supported by Samsung Science and Technology Foundation under Project Number SSTF-BA1701-04.

\section{Semi-Stable Vector Bundles Whose Symmetric Square is Not Stable}

2.1. Unramified Finite Coverings and Prym Varieties. As mentioned in the introduction, the strict semi-stability of $S^{k} E$ has a lot to do with the unramified $k$-coverings of $C$. In this subsection, we review the theory of unramified finite coverings. The references are 12, Exercise III.10.3 \& Exercise IV.2.6] and [3, Chapter 12].

Let $C$ be a smooth projective curve. We will denote by

- $\operatorname{Pic}(C)$ the Picard group of $C$,

- $J^{n}(C)$ the Jacobian of line bundles on $C$ of degree $n$, and

- $J_{m}(C)$ the set of line bundles on $C$ of order $m$; the elements $L \in J^{0}(C)$ with $L^{m}=\mathcal{O}_{C}$.

We will also abuse notation to take a divisor $\mathfrak{b}$ from the group of divisors as $\mathfrak{b} \in \operatorname{Pic}(C)$ instead of $\operatorname{Div}(C)$. 
Let $D$ be an unramified $k$-covering of $C$, that is, there is a finite surjective morphism $\pi: D \rightarrow C$ of degree $k$ whose ramification divisor is empty. Then $\pi^{*}: \operatorname{Pic}(C) \rightarrow \operatorname{Pic}(D)$ induces $\pi^{*}: J^{n}(C) \rightarrow J^{k n}(D)$ since $\operatorname{deg} \pi^{*} L=k \operatorname{deg} L$ for $L \in \operatorname{Pic}(C)$. As $\pi$ is unramified, we have $\omega_{D}=\pi^{*} \omega_{C}$. That is, $\omega_{D / C}=\mathcal{O}_{D}$. Moreover, $\pi^{*}$ has nontrivial kernel; $\pi^{*} L=\mathcal{O}_{D}$ for some $L \in J^{0}(C) \backslash\left\{\mathcal{O}_{C}\right\}$ if and only if $\pi$ is factored as $\pi: D \rightarrow C^{\prime} \stackrel{\eta}{\rightarrow} C$ for some unramified cyclic covering $\eta: C^{\prime} \rightarrow C$ satisfying $\eta^{*} L=\mathcal{O}_{C^{\prime}}$ [3 , Proposition 11.4.3]. Recall that a torsion line bundle $L \in J_{m}(C)$ defines an unramified cyclic $m$-covering $\eta: C^{\prime} \rightarrow C$ and vice versa under the relations $\eta^{*} L=\mathcal{O}_{C^{\prime}}$ and $\eta_{*} \mathcal{O}_{C^{\prime}}=\mathcal{O}_{C} \oplus L^{-1} \oplus \cdots \oplus L^{-(m-1)}$.

To a finite covering $\pi: D \rightarrow C$ of degree $k$, we associate the Norm map $\operatorname{Nm}_{D / C}: \operatorname{Pic}(D) \rightarrow \operatorname{Pic}(C)$ which is defined by

$$
\mathrm{Nm}_{D / C}\left(\sum a_{i} x_{i}\right)=\sum a_{i} \pi\left(x_{i}\right)
$$

in terms of Weil divisors. Then $\mathrm{Nm}_{D / C}$ is a group homomorphism and $\mathrm{Nm}_{D / C} \circ \pi^{*}: \operatorname{Pic}(C) \rightarrow \operatorname{Pic}(C)$ is multiplication by $k$. For $R \in \operatorname{Pic}(D), \pi_{*} R$ is a vector bundle of rank $k$ whose determinant is given by

$$
\operatorname{det} \pi_{*} R \cong \operatorname{det} \pi_{*} \mathcal{O}_{D} \otimes \mathrm{Nm}_{D / C}(R) .
$$

Notice that $\left(\operatorname{det} \pi_{*} \mathcal{O}_{D}\right)^{2}=\mathcal{O}_{C}(-B)$ for the branch divisor $B$ of $\pi: D \rightarrow C$. Thus, if $\pi$ is unramified, then $\left(\operatorname{det} \pi_{*} \mathcal{O}_{D}\right)^{2}=\mathcal{O}_{C}$.

If $V$ is a vector bundle on $C$, then $\pi^{*} V$ is semi-stable if and only if $V$ is semi-stable [14, II: Lemma 6.4.12]. Meanwhile, if $W$ is a vector bundle on $D$, then it is known that $\pi_{*} W$ is stable for general $W$ when $\pi$ is unramified [2]. The following proposition tells that $\pi_{*} W$ is semi-stable and which $\pi_{*} W$ is stable in the case where $\operatorname{deg} \pi=2$ and $\operatorname{rk} W=1$. Notice that any unramified double covering is a cyclic covering.

Proposition 2.1. Let $\pi: B \rightarrow C$ be a nontrivial unramified double covering corresponding to $M \in J_{2}(C)$ and $R \in J^{0}(B)$. Then $E=\pi_{*} R$ is semi-stable and is strictly semi-stable if and only if $R \in \pi^{*} J^{0}(C)$. Moreover, $E$ splits as $E=L \oplus(L \otimes M)$ for some $L \in J^{0}(C)$ when it is strictly semi-stable.

Proof. Note that the natural morphism $\pi^{*} \pi_{*} R \rightarrow R$ becomes surjective because $\pi$ is an affine morphism. Since the kernel of a surjection between vector bundles is a vector bundle, the sequence

$$
0 \rightarrow K \rightarrow \pi^{*} E \rightarrow R \rightarrow 0
$$

is exact for some vector bundle $K$ on $B$ where $\operatorname{rk} K=1$ as $\operatorname{rk} \pi^{*} \pi_{*} R=\operatorname{rk} \pi_{*} R=2$ and $\operatorname{rk} R=1$ in this case. By comparing the determinants using $\operatorname{det} \pi^{*} E=\pi^{*} \operatorname{det} E$, the exact sequence becomes

$$
0 \rightarrow R^{-1} \otimes \pi^{*} \operatorname{det} E \rightarrow \pi^{*} E \rightarrow R \rightarrow 0 \text {. }
$$

Then, from

$$
\operatorname{deg} E=\operatorname{deg}\left(\operatorname{det} \pi_{*} R\right)=\operatorname{deg}\left(\operatorname{det} \pi_{*} \mathcal{O}_{B} \otimes \operatorname{Nm}_{D / C}(R)\right)=\operatorname{deg}\left(\operatorname{det}\left(\mathcal{O}_{C} \oplus M\right) \otimes \operatorname{Nm}_{D / C}(R)\right)=0,
$$

we have $\operatorname{deg} \pi^{*} E=2 \operatorname{deg} E=0$ and $\operatorname{deg}\left(R^{-1} \otimes \pi^{*} \operatorname{det} E\right)=\operatorname{deg} \pi^{*} E-\operatorname{deg} R=0$.

If there is an injection $L \rightarrow E$ for some line bundle $L$ of degree $d$, then we get a nonzero morphism $\pi^{*} L \rightarrow R$ due to the adjoint property, $\operatorname{Hom}\left(\pi^{*} L, R\right)=\operatorname{Hom}\left(L, \pi_{*} R\right)=\operatorname{Hom}(L, E) . \operatorname{As} \operatorname{deg} \pi^{*} L=2 d$, $d \leq 0$ and the equality holds if and only if $R=\pi^{*} L$. Therefore, $E$ is semi-stable, and $E$ is strictly semi-stable if and only if $R \in \pi^{*} J^{0}(C)$.

Now if $E$ is strictly semi-stable so that $R=\pi^{*} L$ for some $L \in J^{0}(C)$, then, by the projection formula,

$$
\pi_{*} R=\pi_{*} \pi^{*} L=\left(\pi_{*} \mathcal{O}_{B}\right) \otimes L=\left(\mathcal{O}_{C} \oplus M\right) \otimes L=L \oplus(L \otimes M) .
$$


In the case where $\pi: B \rightarrow C$ is a nontrivial unramified double covering, we denote the kernel of $\mathrm{Nm}_{B / C}$ by $\operatorname{Pr}(B / C)$ in this paper. Then $\operatorname{Pr}(B / C) \subseteq J^{0}(C)$ and it has two components as

$$
\operatorname{Pr}(B / C)=\left\{S \otimes\left(\iota^{*} S\right)^{-1} \mid S \in \operatorname{Pic}(B)\right\}=\left\{S \otimes\left(\iota^{*} S\right)^{-1} \mid S \in J^{0}(B)\right\} \cup\left\{S \otimes\left(\iota^{*} S\right)^{-1} \mid S \in J^{1}(B)\right\}
$$

where $\iota: B \rightarrow B$ is the involution induced by $\pi$ [16, Lemma 1]. We denote the first summand by $\operatorname{Pr}^{0}(B / C)$ and the second one by $\operatorname{Pr}^{1}(B / C) \cdot \operatorname{Pr}^{0}(B / C)$ is known as the Prym variety of $B$ over $C$ which is an abelian subvariety of $J^{0}(B)$ of dimension $g(B)-g(C)=g-1$, and $\operatorname{Pr}^{1}(B / C)$ is a translate of $\operatorname{Pr}^{0}(C)$ in $J^{0}(B)$. It is also known that $J^{0}(B)=\operatorname{Pr}^{0}(B / C) \otimes \pi^{*} J^{0}(C)=\left\{R \otimes \pi^{*} L \mid R \in \operatorname{Pr}^{0}(B / C), L \in J^{0}(C)\right\}$, and we can describe the intersection $\operatorname{Pr}^{0}(B / C) \cap \pi^{*} J^{0}(C)=\operatorname{Pr}^{0}(B / C) \cap J_{2}(B)$ using the following proposition.

Proposition 2.2. Let $\pi: B \rightarrow C$ be a nontrivial unramified double covering. Then

$$
\operatorname{Pr}(B / C) \cap \pi^{*} J^{0}(C)=\pi^{*} J_{2}(C)=\operatorname{Pr}(B / C) \cap J_{2}(B) .
$$

Proof. As $\operatorname{Nm}_{B / C}\left(\pi^{*} L\right)=L^{2}$ for $L \in \operatorname{Pic}(C), \pi^{*} L \in \operatorname{Pr}(B / C)$ if and only if $L \in J_{2}(C)$. Thus we get $\operatorname{Pr}(B / C) \cap \pi^{*} J^{0}(C)=\pi^{*} J_{2}(C)$, and its order is $2^{2 g-1}$ because $\left|J_{2}(C)\right|=2^{2 g}$ and $\left|\operatorname{ker} \pi^{*}\right|=2$ for the genus $g$ of $C$. From $\pi^{*} J_{2}(C) \subseteq J_{2}(B)$, we have $\pi^{*} J_{2}(C)=\operatorname{Pr}(B / C) \cap \pi^{*} J_{2}(C) \subseteq \operatorname{Pr}(B / C) \cap J_{2}(B)$ and the inclusion becomes equality after calculating the order $\left|\operatorname{Pr}(B / C) \cap J_{2}(B)\right|=2^{2 g-1}$.

Since $\operatorname{Pr}^{0}(B / C)$ is an abelian subvariety of $J^{0}(B)$ of dimension $g-1,\left|\operatorname{Pr}^{0}(B / C) \cap J_{2}(B)\right|=2^{2(g-1)}$. So there exists $L_{1} \in J_{2}(C)$ with $\pi^{*} L_{1} \in \operatorname{Pr}^{1}(B / C)$ but $\pi^{*} L_{1} \notin \operatorname{Pr}^{0}(B / C)$. Then, using the translation $\operatorname{Pr}^{1}(B / C)=\operatorname{Pr}^{0}(B / C) \otimes \pi^{*} L_{1}$, we can deduce that $\left|\operatorname{Pr}^{1}(B / C) \cap J_{2}(B)\right|=2^{2(g-1)}$. Hence we obtain $\left|\operatorname{Pr}(B / C) \cap J_{2}(B)\right|=2^{2 g-1}$ and the equality $\pi^{*} J_{2}(C)=\operatorname{Pr}(B / C) \cap J_{2}(B)$.

2.2. Classification of Orthogonal Bundles. The orthogonal bundles are studied by several authors; Ramanathan [20, Mumford [16], Ramanan [19], Hitching [13], and Biswas-Gómez [5] for instance. In this paper, we use the following definition presented in Hitching [13, which is also similar to that given in Biswas-Gómez [5].

Definition. Let $E$ be a vector bundle and $M$ be a line bundle on $C$. Then $E$ is said to be orthogonal with values in $M$ if there is a nondegenerate symmetric bilinear form $E \otimes E \rightarrow M$.

Let $E$ be a vector bundle on $C$ of rank 2 and degree 0 . If $S^{2} E$ is strictly semi-stable, then there is a quotient line bundle $S^{2} E \rightarrow M$ of $\operatorname{deg} M=0$, which gives a nonzero morphism $E \rightarrow E^{\vee} \otimes M$ from

$$
\operatorname{Hom}\left(S^{2} E, M\right) \subseteq \operatorname{Hom}(E \otimes E, M) \cong H^{0}\left(E^{\vee} \otimes E^{\vee} \otimes M\right) \cong \operatorname{Hom}\left(E, E^{\vee} \otimes M\right) .
$$

If $E$ is stable, then the morphism is necessarily an isomorphism, and the induced symmetric bilinear form $E \otimes E \rightarrow M$ is nondegenerate on each fiber. Hence $E$ admits an orthogonal structure.

Conversely, if $E$ is an orthogonal bundle with values in $M$, then it associates a morphism $S^{2} E \rightarrow M$ which must be surjective because the form $E \otimes E \rightarrow M$ is nondegenerate.

Remark 2.3. Let $E$ be a stable vector bundle on $C$ of rank 2 and degree 0 . Then $E$ is orthogonal if and only if $S^{2} E$ is strictly semi-stable. If $S^{2} E$ is destabilized by a quotient line bundle $S^{2} E \rightarrow M$, then $E$ is orthogonal with values in $M$. Also, by comparing the determinants in the isomorphism $E \cong E^{\vee} \otimes M$, we have $M^{2} \cong(\operatorname{det} E)^{2}$. On the other hand, if $S^{2} E$ is destabilized by a line subbundle $M^{-1} \rightarrow S^{2} E$, then $M^{2}=(\operatorname{det} E)^{-2}$ follows from the isomorphism $E^{\vee} \cong E \otimes M$. In particular, if $E$ is orthogonal with values in $M$ and $E$ has trivial determinant, then $M \in J_{2}(C)$. 
By Mumford's classification [16], if $E$ is an orthogonal bundle of rank 2 with values in $\mathcal{O}_{C}$, then

(1) $E=L^{-1} \oplus L$ for some line bundle $L$, or

(2) $E=\pi_{*} R$ where $\pi: B \rightarrow C$ is an unramified double covering and $R$ is a line bundle on $B$ such that $\operatorname{Nm}_{B / C}(R)=\mathcal{O}_{C}$.

In (2), if $\pi$ is the trivial double covering, then $E=\pi_{*} R$ is the direct sum of line bundles of degree 0 , and it has trivial determinant as $\operatorname{det} \pi_{*} \mathcal{O}_{B}=\operatorname{det}\left(\mathcal{O}_{C} \oplus \mathcal{O}_{C}\right)=\mathcal{O}_{C}$. So $E=L^{-1} \oplus L$ for some $L \in J^{0}(C)$. This case is covered by (1). Otherwise, if $\pi$ is a nontrivial double covering corresponding to $M \in J_{2}(C) \backslash\left\{\mathcal{O}_{C}\right\}$, then we know from Proposition 2.1 that $E=\pi_{*} R$ is semi-stable, and it is strictly semi-stable if and only if $E=L \oplus(L \otimes M)$ for some $L \in J^{0}(C)$. In this case, $L$ must satisfy $L^{2}=\mathcal{O}_{C}$ as $\operatorname{det} E=M$.

Remark 2.4. If $E$ is strictly semi-stable, then $E$ is orthogonal with values in $\mathcal{O}_{C}$ if and only if

- $E=L^{-1} \oplus L$ if the determinant of $E$ is trivial,

- $E=L \oplus(L \otimes M)$ for some $L \in J_{2}(C)$ if $M=\operatorname{det} E$ is nontrivial.

In particular, there is no stable $E$ with trivial determinant whose orthogonal form takes its values in $\mathcal{O}_{C}$.

If $E$ is strictly semi-stable, then $S^{2} E$ is always strictly semi-stable because a surjection $E \rightarrow L$ induces a surjection $S^{2} E \rightarrow L^{2}$. In the same way, if $E$ is not stable, then $S^{k} E$ is not stable for any $k \geq 2$.

We denote by $\mathcal{S U}_{C}\left(2, \mathcal{O}_{C}\right)$ the space of S-equivalence classes of semi-stable vector bundles on $C$ of rank 2 with trivial determinant. After choices of a nontrivial unramified double covering $\pi: B \rightarrow C$ corresponding to $M \in J_{2}(C) \backslash\left\{\mathcal{O}_{C}\right\}$ and a line bundle $A$ satisfying $A^{2}=M$, we can define a map $\Phi_{A}: \operatorname{Pr}(B / C) \rightarrow \mathcal{S U}_{C}\left(2, \mathcal{O}_{C}\right) ; R \mapsto \pi_{*} R \otimes A$ as $\pi_{*} R$ is semi-stable by Proposition 2.1. For fixed $M$, the images of $\Phi_{A}$ are the same under the changes of $A$ because the choices differ by a twist of $L \in J_{2}(C)$ and $\operatorname{Pr}(B / C)$ is invariant under the translation by $\pi^{*} L$ if $L \in J_{2}(C)$.

Proposition 2.5. If $E \in \mathcal{S U}_{C}\left(2, \mathcal{O}_{C}\right)$ is stable and $S^{2} E$ is strictly semi-stable, then $E=\Phi_{A}(R)=$ $\pi_{*} R \otimes A=\pi_{*}\left(R \otimes \pi^{*} A\right)$ for some $\pi: B \rightarrow C, A \in J^{0}(C)$, and $R \in \operatorname{Pr}(B / C)$ as above. Moreover, the locus of $E \in \mathcal{S U}_{C}\left(2, \mathcal{O}_{C}\right)$ where $E$ is stable and $S^{2} E$ is strictly semi-stable has dimension $g-1$.

Proof. Due to Remark 2.3. if $S^{2} E$ is not stable, then $E$ is orthogonal with values in $M \in J_{2}(C) \backslash\left\{\mathcal{O}_{C}\right\}$. Thus for any line bundle $A$ with $A^{2}=M, E \otimes A$ becomes an orthogonal bundle with values in $\mathcal{O}_{C}$. Then, by Mumford's classification, $E \otimes A=\pi_{*} R$ for some nontrivial unramified double covering $\pi: B \rightarrow C$ and $R \in \operatorname{Pr}(B / C)$ where $\pi$ corresponds to $M$ since $\operatorname{det} \pi_{*} \mathcal{O}_{B}=\operatorname{det} \pi_{*} R=A^{2}=M$.

In order to prove the next claim, it is enough to show that $\pi_{*}: \operatorname{Pr}(B / C) \rightarrow \mathcal{S U}_{C}(2, M)$ is generically 2-to-1 for a fixed nontrivial unramified double covering $\pi: B \rightarrow C$ corresponding to $M \in J_{2}(C) \backslash\left\{\mathcal{O}_{C}\right\}$ because $\operatorname{dim} \operatorname{Pr}(B / C)=g-1$ and the choice of $M$ is finite. Let $R, R^{\prime} \in \operatorname{Pr}(B / C)$ and $E=\pi_{*} R=\pi_{*} R^{\prime}$. As $\pi^{*} \operatorname{det} E=\pi^{*} M=\mathcal{O}_{B}$, we get the following exact sequences on $B$ (see the proof of Proposition 2.1).

$$
0 \rightarrow R^{-1} \rightarrow \pi^{*} E \rightarrow R \rightarrow 0 \text { and } 0 \rightarrow R^{\prime-1} \rightarrow \pi^{*} E \rightarrow R^{\prime} \rightarrow 0
$$

If $R^{\prime} \neq R$, then there exists a nonzero morphism $R^{-1} \rightarrow R^{\prime}$ by adjoining the morphisms $R^{-1} \rightarrow \pi^{*} E$ and $\pi^{*} E \rightarrow R^{\prime}$. Since both $R$ and $R^{\prime}$ have degree $0, R^{\prime} \cong R^{-1}$. So we have either $R^{\prime}=R$ or $R^{\prime}=R^{-1}$. Here, $R^{-1}=\iota^{*} R$ for the involution $\iota: B \rightarrow B$ induced by $\pi$ because $R=S \otimes\left(\iota^{*} S\right)^{-1}$ for some $S \in \operatorname{Pic}(B)$. Note that $R=\iota^{*} R$ if and only if $R \in \pi^{*} J^{0}(C)$, and $\operatorname{Pr}(B / C) \cap \pi^{*} J^{0}(C)$ is finite by Proposition 2.2.

Recall that the dimension of the moduli $\mathcal{S U}_{C}\left(2, \mathcal{O}_{C}\right)$ is $3 g-3$, and the locus of $E \in \mathcal{S U}_{C}\left(2, \mathcal{O}_{C}\right)$ with $S^{2} E$ being strictly semi-stable is the union of the above locus and the locus of strictly semi-stable $E$. The latter locus is given by the image of $J^{0}(C) \rightarrow \mathcal{S U}_{C}\left(2, \mathcal{O}_{C}\right) ; L \mapsto\left[L^{-1} \oplus L\right]$, and its dimension is $g$. 
2.3. $k$-Sections on a Ruled Surface. The material of this and the next subsection is well-known, but we include it for the sake of notational clarity. Let $E$ be a vector bundle on $C$ of rank 2 . The projective space bundle $X=\mathbb{P}_{C}(E)$ with projection $\Pi: X \rightarrow C$ is called a ruled surface over $C$. We choose the convention that $\mathbb{P}_{C}(E)$ is regarded as the projective space of lines in the fibers. By Tsen's theorem, there exists a section of $\Pi$ and it is possible to regard the image as an effective divisor $C \subseteq X$. The Picard group of $X$ is given by $\operatorname{Pic}(X)=\mathbb{Z} C \oplus \Pi^{*} \operatorname{Pic}(C)=\{k C+\mathfrak{b} f \mid k \in \mathbb{Z}, \mathfrak{b} \in \operatorname{Pic}(C)\}$. A k-secant divisor is a divisor $D$ on $X$ linearly equivalent to $k C+\mathfrak{b} f$ for some $\mathfrak{b} \in \operatorname{Pic}(C)$, and is called a $k$-section if $D$ is effective. If $k=1$ (resp. 2,3 ), then a $k$-section $D$ is said to be a section (resp. bisection, trisection). We will denote linear equivalence by $\sim$ and numerical equivalence by $\equiv$.

We fix a unisecant divisor $C_{1}$ on $X$ which satisfies $\pi_{*} \mathcal{O}_{X}\left(C_{1}\right)=E$. Then $\pi_{*} \mathcal{O}_{X}\left(k C_{1}\right)=S^{k} E$ for $k \geq 0$, $\pi_{*} \mathcal{O}_{X}\left(k C_{1}\right)=0$ for $k<0$, and $R^{1} \pi_{*} \mathcal{O}_{X}\left(k C_{1}\right)=\pi_{*} \mathcal{O}_{X}\left(-(k+2) C_{1}\right)^{\vee} \otimes(\operatorname{det} E)^{\vee}$. We can also deduce that $C_{1}{ }^{2}=\operatorname{deg} E$. There is a correspondence between $k$-sections on $X$ and line subbundles of $S^{k} E$ given by effective $D \sim k C_{1}+\mathfrak{b} f \leftrightarrow D \in H^{0}\left(\mathcal{O}_{X}\left(k C_{1}+\mathfrak{b} f\right)\right) \leftrightarrow s \in H^{0}\left(S^{k} E \otimes L\right) \leftrightarrow$ inclusion $L^{-1} \stackrel{s}{\rightarrow} S^{k} E$ for $L=\mathcal{O}_{C}(\mathfrak{b})$, and the self-intersection number of $D$ is equal to

$$
D^{2}=\left(k C_{1}+\mathfrak{b} f\right)^{2}=k^{2} C_{1}^{2}+2 k \operatorname{deg} \mathfrak{b}=k^{2} \operatorname{deg} E+2 k \operatorname{deg} L .
$$

For $k=1$, a section $C_{0}$ is called a minimal section if it attains the minimal self-intersection number $D^{2}$ among the sections $D \sim C_{1}+\mathfrak{b} f$ for some $\mathfrak{b} \in \operatorname{Pic}(C)$. Though the choice of $C_{0}$ may not be unique, but the number $C_{0}{ }^{2}$ is uniquely determined by $E$, and it is called the Segre invariant $s_{1}(E)$. From the definition of stability, it is easy to check that $E$ is stable (resp. semi-stable) if and only if ${C_{0}}^{2}>0$ (resp. $C_{0}{ }^{2} \geq 0$ ).

Let $E$ be a semi-stable vector bundle on $C$ of rank 2 and degree 0 . Then $S^{k} E$ is a semi-stable vector bundle of degree 0 , and if $L^{-1} \rightarrow S^{k} E$ is a line subbundle of $\operatorname{deg} L=b$, then it must follow that $b \geq 0$. That is, $D \equiv k C_{1}+b f$ is effective only if $b \geq 0$. Thus the cone of curves $\mathrm{NE}(X) \subseteq N_{1}(X)$ is contained in the cone $\mathcal{C} \subseteq N_{1}(X)$ which is $\mathbb{R}_{\geq 0}$-spanned by the rays $[f]$ and $\left[C_{1}\right]$. It is further possible to show that $\overline{\mathrm{NE}}(X)=\mathcal{C}$ when $E$ is semi-stable [14, I: p. 70]. Therefore, $S^{k} E$ is destabilized by a line subbundle if there exists a $k$-section $D \equiv k C_{1}$ on $X$ for some $k>0$, which is equivalent to saying that $\operatorname{NE}(X)$ is closed.

Remark 2.6. Let $E$ be a semi-stable vector bundle on $C$ of rank 2 with even degree. There are various characterizations of a $k$-section $D$ on $X$ which corresponds to a destabilizing line subbundle $L^{-1} \rightarrow S^{k} E$.

(1) $D$ has zero self-intersection.

(2) $D$ lies on the boundary of $\mathrm{NE}(X)$.

(3) $\pi=\left.\Pi\right|_{D}: D \rightarrow C$ is an unramified $k$-covering if $D$ is irreducible and reduced.

(4) $D$ is a smooth curve of genus $k g-k+1$ where $g$ is the genus of $C$ if $D$ is irreducible and reduced. The proof of (3) is introduced in Rosoff [21, p. 123: the first remark]. By (3), D is smooth, so the other equivalences can be shown using the adjunction and Hurwitz formula.

Proposition 2.7. Let $E$ be a vector bundle on $C$ of rank 2 and degree 0 . Let $X=\mathbb{P}_{C}(E)$ be the ruled surface $\Pi: \mathbb{P}_{C}(E) \rightarrow C$ and $C_{1}$ be a unisecant divisor on $X$ with $\Pi_{*} \mathcal{O}_{X}\left(C_{1}\right) \cong E$. If there is an irreducible and reduced $k$-section $D \sim k C_{1}+\mathfrak{b} f$ for some $\mathfrak{b} \in \operatorname{Pic}(C)$ of $\operatorname{deg} \mathfrak{b}=0$, then $\mathcal{O}_{D}\left((k-2) C_{1}+\mathfrak{b} f\right)=\mathcal{O}_{D}$.

Proof. By Remark 2.6. $D$ is smooth. Then we can use the adjunction formula,

$$
\omega_{D}=\left.\mathcal{O}_{X}\left(K_{X}+D\right)\right|_{D}=\left.\mathcal{O}_{X}\left(\left(-2 C_{1}+K_{C} f\right)+\left(k C_{1}+\mathfrak{b} f\right)\right)\right|_{D},
$$

and it shows that $\omega_{D / C}=\mathcal{O}_{D}\left((k-2) C_{1}+\mathfrak{b} f\right)$. Again by Remark 2.6, $D$ is unramified over $C$ so that $\omega_{D / C}=\mathcal{O}_{D}$. Thus we have $\mathcal{O}_{D}\left((k-2) C_{1}+\mathfrak{b} f\right)=\mathcal{O}_{D}$. 
2.4. Elementary Transformations. Let $E$ be a vector bundle on $C$ of rank 2 and $X=\mathbb{P}_{C}(E)$ be the ruled surface $\Pi: \mathbb{P}_{C}(E) \rightarrow C$. There are two notions of elementary transformations. One is for vector bundles and the other is for ruled surfaces. First, we explain the elementary transformation of vector bundles. Let $P \in C$ and fix a line $x$ in the fiber $\left.E\right|_{P}$. The elementary transformation $\operatorname{elm}_{x} E$ of $E$ at $x$ is defined by the following exact sequence.

$$
0 \rightarrow \operatorname{elm}_{x} E \rightarrow E \stackrel{\alpha(x)}{\longrightarrow} \mathbb{C}_{P} \rightarrow 0
$$

Here, $\alpha(x)$ has the kernel $x$ at the fiber $\left.E\right|_{P} \rightarrow \mathbb{C}_{P}$. Note that $\operatorname{det}(\operatorname{elm} x E)=\operatorname{det} E \otimes \mathcal{O}_{C}(-P)$.

Next, for the elementary transformation of ruled surfaces, let $x \in X$ be a closed point. Notice that the point $x$ in the fiber $P f=\Pi^{-1}(P)$ can be identified with a line in the fiber $\left.E\right|_{P}$ over $P=\Pi(x)$. Then the elementary transformation $Y:=\operatorname{elm}_{x} X$ of $X$ at $x$ is the surface given by the following process.

(1) $\widetilde{X}$ is the blow-up of $X$ at $x$. The strict transform $\widetilde{P f} \subseteq \widetilde{X}$ of the fiber $P f \subseteq X$ is a (-1)-curve.

(2) $Y$ is the blow-down of $\widetilde{X}$ along $\widetilde{P f}$. Then, the strict transform $Z^{\prime} \subseteq Y$ of the exceptional divisor $Z \subseteq \tilde{X}$ for $\tilde{X} \rightarrow X$ becomes a smooth rational curve of zero self-intersection.

Then $Y$ is again a ruled surface $\Lambda: \mathbb{P}_{C}(F) \rightarrow C$ for some vector bundle $F$ on $C$ of rank 2. We denote the blow-up and down by $\varphi: \widetilde{X} \rightarrow X$ and $\psi: \widetilde{X} \rightarrow Y$, and the center of the blow-down $\psi$ by $y \in Y$.

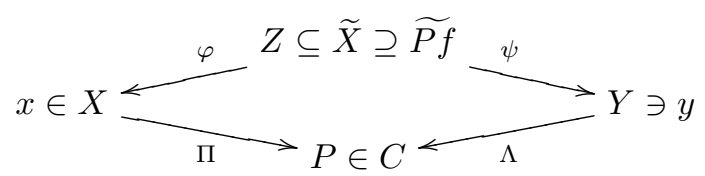

Let $C_{1}$ be a unisecant divisor on $X$ such that $E=\Pi_{*} \mathcal{O}_{X}\left(C_{1}\right)$. Then, for a section $D \sim C_{1}+\mathfrak{b} f$ on $X$, we have $\Pi_{*} \mathcal{O}_{X}(D)=E \otimes L$ for $L=\mathcal{O}_{C}(\mathfrak{b})$. We will see the nature of the strict transform $D^{\prime} \subseteq Y$ of $D \subseteq X$ after the elementary transformation with regard to either $x \in D \subseteq X$ or $x \notin D \subseteq X$. Let $F=\Lambda_{*} \mathcal{O}_{Y}\left(D^{\prime}\right)$. If $x \in D \subseteq X$, then $y \notin D^{\prime} \subseteq Y$, and

$$
\begin{aligned}
F & =\Lambda_{*} \mathcal{O}_{Y}\left(D^{\prime}\right)=\Lambda_{*}\left(\psi_{*}\left(\psi^{*} \mathcal{O}_{Y}\left(D^{\prime}\right)\right)\right) \\
& =(\Lambda \circ \psi)_{*}\left(\mathcal{O}_{\widetilde{X}}(\widetilde{D})\right)=(\Pi \circ \phi)_{*}\left(\mathcal{O}_{\widetilde{X}}(\widetilde{D}+Z) \otimes \mathcal{O}_{\widetilde{X}}(-Z)\right) \\
& =\Pi_{*}\left(\phi_{*}\left(\phi^{*} \mathcal{O}_{X}(D) \otimes \mathcal{O}_{\widetilde{X}}(-Z)\right)\right)=\Pi_{*}\left(\mathcal{O}_{X}(D) \otimes \mathcal{I}_{x}\right)
\end{aligned}
$$

where $\widetilde{D} \subseteq \widetilde{X}$ is the strict transform of $D \subseteq X$. On the other hand, consider the exact sequence

$$
0 \rightarrow \mathcal{O}_{X}(D) \otimes \mathcal{I}_{x} \rightarrow \mathcal{O}_{X}(D) \rightarrow \mathbb{C}_{x} \rightarrow 0
$$

on $X$. By pushing forward the sequence, we obtain the following exact sequence on $C$.

$$
0 \rightarrow F \rightarrow E \otimes L \stackrel{\beta}{\rightarrow} \mathbb{C}_{P} \rightarrow 0
$$

As $\beta=\alpha(x)$, it shows that $F=\operatorname{elm}_{x}(E \otimes L)=\operatorname{elm}_{x} E \otimes L$. Next, if $x \notin D \subseteq X$, then $y \in D^{\prime} \subseteq Y$, and

$$
\begin{aligned}
F & =\Lambda_{*} \mathcal{O}_{Y}\left(D^{\prime}\right)=\Lambda_{*}\left(\psi_{*}\left(\psi^{*} \mathcal{O}_{Y}\left(D^{\prime}\right)\right)\right) \\
& =(\Lambda \circ \psi)_{*}\left(\mathcal{O}_{\widetilde{X}}(\widetilde{D}+\widetilde{P f})\right)=(\Pi \circ \phi)_{*}\left(\mathcal{O}_{\widetilde{X}}(\widetilde{D}+\widetilde{P f}+Z) \otimes \mathcal{O}_{\widetilde{X}}(-Z)\right) \\
& =\Pi_{*}\left(\phi_{*}\left(\phi^{*} \mathcal{O}_{X}(D+P f) \otimes \mathcal{O}_{\widetilde{X}}(-Z)\right)\right)=\Pi_{*}\left(\mathcal{O}_{X}(D) \otimes \mathcal{I}_{x}\right) \otimes \mathcal{O}_{C}(P)
\end{aligned}
$$

where $\widetilde{P f} \subseteq \widetilde{X}$ and $\widetilde{D} \subseteq \widetilde{X}$ are as before. By the same argument, we get the exact sequence

$$
0 \rightarrow F \otimes \mathcal{O}_{C}(-P) \rightarrow E \otimes L \stackrel{\beta}{\rightarrow} \mathbb{C}_{P} \rightarrow 0
$$

on $C$. Because $\beta=\alpha(x)$, we deduce that $F=\operatorname{elm}_{x}(E \otimes L) \otimes \mathcal{O}_{C}(P)=\operatorname{elm}_{x} E \otimes L(P)$. 
Proposition 2.8. Let $D$ be the section on $X=\mathbb{P}_{C}(E)$ corresponding to a line subbundle $L^{-1} \rightarrow E$. Then, for the elementary transformation $Y=\operatorname{elm}_{x} X$ of $X$ at a point $x$ over $P \in C$, and the strict transform $D^{\prime} \subseteq Y$ of $D \subseteq X$, there exists the corresponding line subbundle

$$
\begin{cases}L^{-1} \rightarrow \operatorname{elm}_{x} E \text { to } D^{\prime} \subseteq Y \text { and }\left(D^{\prime}\right)^{2}=D^{2}-1 & \text { if } x \in D \subseteq X, \\ L^{-1}(-P) \rightarrow \operatorname{elm}_{x} E \text { to } D^{\prime} \subseteq Y \text { and }\left(D^{\prime}\right)^{2}=D^{2}+1 & \text { if } x \notin D \subseteq X .\end{cases}
$$

Proof. Note that, in the case where $x \in D$, the strict transform $D^{\prime}$ on $Y$ corresponds to the line subbundle $L^{-1} \rightarrow \operatorname{elm}_{x} E$. So the self-intersection number is given by

$$
\left(D^{\prime}\right)^{2}=\operatorname{deg}(\operatorname{elm} x E)+2 \operatorname{deg} L=(\operatorname{deg} E-1)+2 \operatorname{deg} L=(\operatorname{deg} E+2 \operatorname{deg} L)-1=D^{2}-1 .
$$

The proof is similar for the case where $x \notin D$.

In the both cases, whether $x \in D$ or not, we can see that $\operatorname{elm}_{x}\left(\Pi_{*} \mathcal{O}_{X}(D)\right)$ and $\Lambda_{*} \mathcal{O}_{Y}\left(D^{\prime}\right)$ differ by a twist of a line bundle, and hence $Y=\mathbb{P}_{C}(F)=\mathbb{P}_{C}\left(\operatorname{elm}_{x} E\right)$ for $Y=\operatorname{elm}_{x} X$.

The elementary transformation can be defined at multiple points of $X$ unless the set of points contains distinct points in the same fiber of $\Pi: X \rightarrow C$. We introduce an example of an elementary transformation taken at a double point $x$ $y$ in which $x$ is a closed point of $X$ over $P \in C$ and $y$ is a point infinitely near to $x$ but not the infinitely near point of the fiber $P f \subseteq X$. Equivalently, $y$ is a closed point of $\operatorname{elm}_{x} X$ over the same point $P \in C$ but not the center of the blow-down $\mathrm{Bl}_{x} X \rightarrow \operatorname{elm}_{x} X$.

Example 2.9. Let $X=\mathbb{P}_{C}(E)$ be a ruled surface $\Pi: \mathbb{P}_{C}(E) \rightarrow C$ and $D$ be a section on $X$ such that $\Pi_{*} \mathcal{O}_{X}(D)=E \otimes L$ for some line bundle $L$. Let $x$ be a closed point of $D$ and $y$ be the infinitely near point of $D$ at $x$ given by the intersection point $\widetilde{D} \cap Z$ of the exceptional fiber $Z$ of the blow-up $\mathrm{Bl}_{x} X \rightarrow X$ and the strict transform $\widetilde{D}$ of $D$ on $\mathrm{Bl}_{x} X$. Let $P=\Pi(x)$ and $\mathcal{I}_{x \text { m } y}$ be the ideal sheaf on $X$ which defines $y$ infinitely near to $x$. By pushing forward the exact sequence

$$
0 \rightarrow \mathcal{O}_{X}(D) \otimes \mathcal{I}_{x \leftrightarrow y} \rightarrow \mathcal{O}_{X}(D) \rightarrow \mathcal{O}_{X} / \mathcal{I}_{x \leftrightarrow y} \rightarrow 0
$$

on $X$, we have the following exact sequence on $C$.

$$
0 \rightarrow \operatorname{elm}_{x+y} E \otimes L \rightarrow E \otimes L \rightarrow \mathcal{O}_{2 P} \rightarrow 0
$$

By Proposition 2.8, we obtain that $\Lambda_{*}\left(\mathcal{O}_{Y}\left(D^{\prime}\right)\right)=\operatorname{elm}_{x \leftarrow y} E \otimes L$ where $Y=\operatorname{elm}_{x \leftarrow y} X$ is a ruled surface $\Lambda: Y \rightarrow C$ and $D^{\prime} \subseteq Y$ is the strict transform of $D \subseteq X$.

2.5. Generation of Orthogonal Bundles by Elementary Transformations. Choi and Park [8] use elementary transformations to construct a ruled surface $X=\mathbb{P}_{C}(E)$ where $E$ is stable and $X$ admits a bisection of zero self-intersection. As we have seen so far, in this case, $S^{2} E$ is strictly semi-stable. Also, $E$ is orthogonal if the degree of $E$ is normalized to be 0 since $E$ is stable. In this subsection, we will briefly review the construction, and show that the elementary transformation construction generates all the orthogonal bundles.

Let $M \in J_{2}(C)$ be nontrivial and $Y$ be the ruled surface $\Lambda: \mathbb{P}_{C}\left(\mathcal{O}_{C} \oplus M\right) \rightarrow C$. Then $Y$ has only two minimal sections $C_{0}$ and $C_{\infty}$ which respectively correspond to $\mathcal{O}_{C} \rightarrow \mathcal{O}_{C} \oplus M$ and $M \rightarrow \mathcal{O}_{C} \oplus M$. Because there is a 1-dimensional family of bisections on $Y$ linearly equivalent to $2 C_{0}$ whereas $Y$ has only finitely many sections numerically equivalent to $C_{0}$, there exists an irreducible bisection in the linear equivalence class of $2 C_{0}$. So fix an irreducible bisection $B^{\prime} \sim 2 C_{0}$ on $Y$ which corresponds to a line 
subbundle $\mathcal{O}_{C} \rightarrow S^{2}\left(\mathcal{O}_{C} \oplus M\right)=\mathcal{O}_{C} \oplus M \oplus \mathcal{O}_{C}$. Then we can obtain the desired ruled surfaces by taking elementary transformations of $Y$ at general points of $B^{\prime}$.

Let $x_{1}, x_{2}, \ldots, x_{2 n}$ be arbitrary closed points of $B^{\prime}$ on $Y$ and $X$ be the ruled surface $\Pi: X \rightarrow C$ obtained by taking elementary transformations of $Y$ at $x_{1}, x_{2}, \ldots, x_{2 n}$. To avoid technical issues, we will not deal with the cases where the points involve distinct closed points in the same fiber of $\Lambda$. However, we allow repeated points. If $x_{1}=x_{2}=\cdots=x_{m}$ for example, then we can take an elementary transformation at $x_{1} \backsim x_{2} \backsim \cdots \backsim x_{m}$ where $x_{i+1}$ is infinitely near to $x_{1}$ in the direction $i$-th tangent to $B^{\prime}$, that is, $x_{i+1}$ is given recursively by the intersection point $\widetilde{B_{i}^{\prime}} \cap Z_{i}$ on the $i$-th blow-up $Y_{i} \rightarrow Y$ where $Z_{i}$ is the exceptional divisor of the blow-up $Y_{i} \rightarrow Y_{i-1}$ centered at $x_{i}$ with initial $Y_{0}=Y$ and $\widetilde{B_{i}^{\prime}} \subseteq Y_{i}$ is the strict transform of $B^{\prime} \subseteq Y$.

Since ${B^{\prime}}^{2}=0$, the smoothness of $B^{\prime}$ follows from Remark 2.6. Then it is easy to check that the strict transform $B \subseteq X$ of $B^{\prime} \subseteq Y$ satisfies $B^{2}=0$ as well. Again by Remark 2.6. $B$ is smooth, so the strict transform from $B^{\prime}$ to $B$ is an isomorphism. Thus we can regard the points $x_{1}, x_{2}, \ldots, x_{2 n}$ as points of $B$, and $\pi=\left.\Pi\right|_{B}: B \rightarrow C$ as the unramified double covering corresponding to $M$ as the same with $B^{\prime} \rightarrow C$.

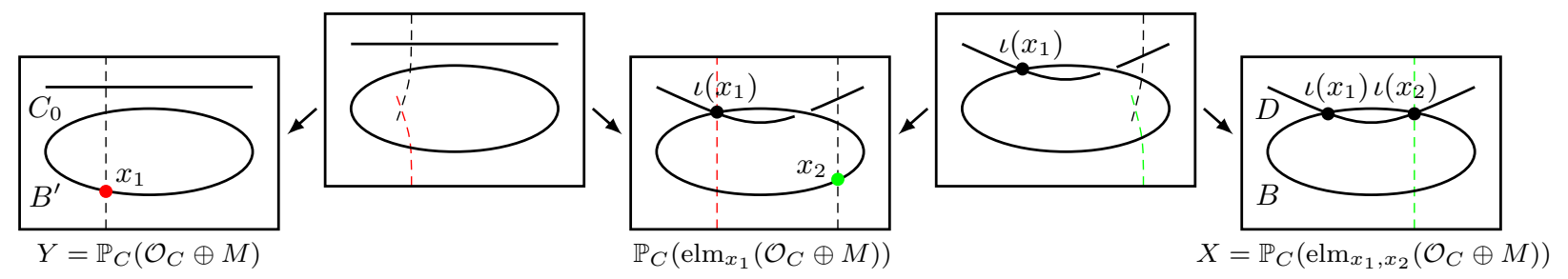

FIGURE 1. Elementary transformation at $2 n$ points for $n=1$

Let $D$ be a section on $X$ given by the strict transform of $C_{0} \subseteq Y$. As we can observe from the diagram of the case $n=1$, we have

$$
\left.\mathcal{O}_{X}(D)\right|_{B}=\mathcal{O}_{B}\left(\iota\left(x_{1}\right)+\iota\left(x_{2}\right)+\cdots+\iota\left(x_{2 n}\right)\right)
$$

where $\iota: B \rightarrow B$ is the involution induced by $\pi$. Let $P_{i}=\Pi\left(x_{i}\right)$ for $i=1,2, \ldots, 2 n$ and $L=\mathcal{O}_{C}(\mathfrak{b})$ be a line bundle on $C$ such that $L^{2}=\mathcal{O}_{C}\left(P_{1}+P_{2}+\cdots+P_{2 n}\right)$. Then

$$
\mathrm{Nm}_{B / C}\left(\mathcal{O}_{B}\left(\iota\left(x_{1}\right)+\iota\left(x_{2}\right)+\cdots+\iota\left(x_{2 n}\right)\right) \otimes \pi^{*} L^{-1}\right)=\mathcal{O}_{C},
$$

and hence $E:=\pi_{*}\left(\mathcal{O}_{B}\left(\iota\left(x_{1}\right)+\iota\left(x_{2}\right)+\cdots+\iota\left(x_{2 n}\right)\right) \otimes \pi^{*} L^{-1}\right)=\pi_{*} \mathcal{O}_{B}(D-\mathfrak{b} f)$ is an orthogonal bundle with values in $\mathcal{O}_{C}$ whose rank is 2 and determinant is $M$. Since $B$ is effective, there exists the exact sequence

$$
\left.0 \rightarrow \mathcal{O}_{X}(D-\mathfrak{b} f-B) \rightarrow \mathcal{O}_{X}(D-\mathfrak{b} f) \rightarrow \mathcal{O}_{X}(D-\mathfrak{b} f)\right|_{B} \rightarrow 0
$$

on $X$, and by pushing forward the sequence to $C$, we get $E=\Pi_{*} \mathcal{O}_{X}(D-\mathfrak{b} f)=\Pi_{*} \mathcal{O}_{X}(D) \otimes L^{-1}$. Applying Proposition 2.8, we have $\operatorname{elm}_{x_{1}, x_{2}, \ldots, x_{2 n}}\left(\mathcal{O}_{C} \oplus M\right)=\Pi_{*} \mathcal{O}_{X}(D) \otimes \mathcal{O}_{C}\left(-P_{1}-P_{2}-\cdots-P_{2 n}\right)$ because none of $x_{i}$ is contained in $C_{0}$ due to $C_{0} . B=0$. Therefore,

$$
E=\left(\Pi_{*} \mathcal{O}_{X}(D) \otimes L^{-2}\right) \otimes L=\operatorname{elm}_{x_{1}, x_{2}, \ldots, x_{2 n}}\left(\mathcal{O}_{C} \oplus M\right) \otimes L .
$$

Thanks to Proposition 2.1 this argument further asserts that $E$ is semi-stable and stable in general. The next theorem shows that this process generates the orthogonal bundles. 
Theorem 2.10. Let $M \in J_{2}(C) \backslash\left\{\mathcal{O}_{C}\right\}$ and $E$ be a vector bundle on $C$ of rank 2 and determinant $M$. If $E$ is orthogonal with values in $\mathcal{O}_{C}$, then there exist points $x_{1}, x_{2} \ldots, x_{2 n}$ of a bisection $B^{\prime}$ on the ruled surface $Y=\mathbb{P}_{C}\left(\mathcal{O}_{C} \oplus M\right)$ such that $E=\operatorname{elm}_{x_{1}, x_{2}, \ldots, x_{2 n}}\left(\mathcal{O}_{C} \oplus M\right) \otimes L$ for some $L \in J^{n}(C)$.

Proof. Let $B^{\prime} \subseteq Y$ be as before and $\pi: B \rightarrow C$ be the unramified double covering corresponding to $M$ with involution $\iota: B \rightarrow B$. Then $E=\pi_{*} R$ for some $R \in \operatorname{Pr}(B / C)$. Fix a line bundle $L$ on $C$ of degree $g$. Since $2 g \geq 2 g-1=g(B)=\operatorname{dim} J^{2 g}(B)$, there exist points $x_{1}, x_{2}, \ldots, x_{2 g} \in B \cong B^{\prime}$ such that

$$
\mathcal{O}_{B}\left(\iota\left(x_{1}\right)+\iota\left(x_{2}\right)+\cdots+\iota\left(x_{2 g}\right)\right)=R \otimes \pi^{*} L \text { in } J^{2 g}(B)
$$

and $P_{i}=\pi\left(x_{i}\right)$ satisfies $L^{2}=\operatorname{Nm}_{B / C}\left(R \otimes \pi^{*} L\right)=\mathcal{O}_{C}\left(P_{1}+P_{2}+\cdots+P_{2 g}\right)$ because $\operatorname{Nm}_{B / C}(R)=\mathcal{O}_{C}$. If there are two points of the form $x$ and $\iota(x)$ in $x_{i}$, say $x_{2 g}=\iota\left(x_{2 g-1}\right)$, then, by taking subtraction as

$$
\mathcal{O}_{B}\left(\iota\left(x_{1}\right)+\iota\left(x_{2}\right)+\cdots+\iota\left(x_{2 g-2}\right)\right)=R \otimes \pi^{*}\left(L\left(-P_{g}\right)\right) \text { in } J^{2 g-2}(B)
$$

we may assume that there is no pair of points in $x_{i}$ of the form $x$ and $\iota(x)$. Thus we can apply the previous argument to have $E \cong \operatorname{elm}_{x_{1}, x_{2}, \ldots, x_{2 n}}\left(\mathcal{O}_{C} \oplus M\right) \otimes L$.

For the completeness of the exposition, we leave the following remark which states that the orthogonal bundles of the form $A^{-1} \oplus A$ are also generated by elementary transformations from $\mathcal{O}_{C} \oplus \mathcal{O}_{C}$.

Remark 2.11. Let $Y$ be the ruled surface $\Lambda: \mathbb{P}_{C}\left(\mathcal{O}_{C} \oplus \mathcal{O}_{C}\right) \rightarrow C$. We can choose two distinct sections $C_{0}$ and $C_{\infty}$ on $Y$ corresponding to different inclusions $\mathcal{O}_{C} \rightarrow \mathcal{O}_{C} \oplus \mathcal{O}_{C}$. Then they have no intersection. For $x_{1}, \ldots, x_{n} \in C_{0}$ and $x_{n+1}, \ldots, x_{2 n} \in C_{\infty}$ with $P_{i}=\Lambda\left(x_{i}\right)$, we can define $\operatorname{elm}_{x_{1}, x_{2}, \ldots, x_{2 n}} F$ whenever $\left\{P_{1}, \ldots, P_{n}\right\} \cap\left\{P_{n+1}, \ldots, P_{2 n}\right\}=\emptyset$. By Proposition 2.8, we have two distinct injections

$\mathcal{O}_{C}\left(-P_{n+1}-\cdots-P_{2 n}\right) \rightarrow \operatorname{elm}_{x_{1}, x_{2}, \ldots, x_{2 n}}\left(\mathcal{O}_{C} \oplus \mathcal{O}_{C}\right)$ and $\mathcal{O}_{C}\left(-P_{1}-\cdots-P_{n}\right) \rightarrow \operatorname{elm}_{x_{1}, x_{2}, \ldots, x_{2 n}}\left(\mathcal{O}_{C} \oplus \mathcal{O}_{C}\right)$.

Since they destabilize $\operatorname{elm}_{x_{1}, x_{2}, \ldots, x_{2 n}}\left(\mathcal{O}_{C} \oplus \mathcal{O}_{C}\right)$, we have

$$
\operatorname{elm}_{x_{1}, x_{2}, \ldots, x_{2 n}}\left(\mathcal{O}_{C} \oplus \mathcal{O}_{C}\right) \otimes L=L\left(-P_{1}-\cdots-P_{n}\right) \oplus L\left(-P_{i+1}-\cdots-P_{2 n}\right),
$$

for any $L \in \operatorname{Pic}(C)$. As the choices of $x_{i}$ are arbitrary, we can generate $E=A^{-1} \oplus A$ for all $A \in J^{0}(C)$ in this way. Indeed, after fixing $L \in J^{g}(C)$, we can find points $P_{1}, P_{2} \ldots, P_{2 g} \in C$ which satisfy $A=L\left(-P_{1}-\cdots-P_{g}\right)$ and $A^{-1}=L\left(-P_{g+1}-\cdots-P_{2 g}\right)$. If $\left\{P_{1}, \ldots, P_{g}\right\} \cap\left\{P_{g+1}, \ldots, P_{2 g}\right\} \neq \emptyset$, say $P_{g}=P_{2 g}$, then we can reduce to the case $A=L^{\prime}\left(-P_{1}-\cdots-P_{g-1}\right)$ and $A^{-1}=L^{\prime}\left(-P_{g+1}-\cdots-P_{2 g-1}\right)$ by substituting $L^{\prime}=L\left(-P_{g}\right)$ for $L$. Thus we can obtain that $\operatorname{elm}_{x_{1}, x_{2}, \ldots, x_{2 n}}\left(\mathcal{O}_{C} \oplus \mathcal{O}_{C}\right) \otimes L=A^{-1} \oplus A$ for some $L \in J^{n}(C)$ after the substitutions.

\section{Semi-Stable Vector Bundles Whose Symmetric Cube is Not Stable}

3.1. Destabilized by Rank 1. Let $E$ be a stable vector bundle on $C$ of rank 2. If $S^{3} E$ is not stable, then it is destabilized by a subbundle of rank 1 or 2 by Remark 1.1. We first study the case of rank 1 .

Proposition 3.1. Let $k \geq 2$. For a vector bundle $E$ of rank 2 and any line bundle $L$ on $C$, there exist the following exact sequences.

$$
\begin{gathered}
0 \rightarrow \operatorname{Hom}\left(S^{k+1} E, L \otimes \operatorname{det} E\right) \rightarrow \operatorname{Hom}\left(S^{k} E, E \otimes L\right) \rightarrow \operatorname{Hom}\left(S^{k-1} E, L\right) \\
0 \rightarrow \operatorname{Hom}\left(L^{-1} \otimes \operatorname{det} E, S^{k+1} E\right) \rightarrow \operatorname{Hom}\left(E \otimes L^{-1}, S^{k} E\right) \rightarrow \operatorname{Hom}\left(L^{-1}, S^{k-1} E\right)
\end{gathered}
$$

In particular, when $E$ is stable and has trivial determinant, for a line bundle $L$ of degree 0 , 
- if $S^{k+1} E$ is destabilized by $S^{k+1} E \rightarrow L$, then $S^{k} E$ is destabilized by $S^{k} E \rightarrow E \otimes L$,

- if $S^{k+1} E$ is destabilized by $L^{-1} \rightarrow S^{k+1} E$, then $S^{k} E$ is destabilized by $E \otimes L^{-1} \rightarrow S^{k} E$,

and the converses hold if $S^{k-2} E$ is stable.

Proof. Let $X=\mathbb{P}_{C}(E)$ be the ruled surface $\Pi: \mathbb{P}_{C}(E) \rightarrow C$ and $C_{1}$ be a unisecant divisor on $X$ satisfying $\Pi_{*} \mathcal{O}_{X}\left(C_{1}\right)=E$. Since the natural morphism $\Pi^{*} E \rightarrow \mathcal{O}_{C}\left(C_{1}\right)$ is surjective, we have the following exact sequence on $X$.

$$
0 \rightarrow \mathcal{O}_{X}\left(-C_{1}\right) \otimes \Pi^{*} \operatorname{det} E \rightarrow \Pi^{*} E \rightarrow \mathcal{O}_{X}\left(C_{1}\right) \rightarrow 0
$$

By pushing forward the sequence after twisting by $\mathcal{O}_{X}\left(k C_{1}\right)$ for $k \geq 0$, we obtain the following exact sequence on $C$.

$$
0 \rightarrow S^{k-1} E \otimes \operatorname{det} E \rightarrow S^{k} E \otimes E \rightarrow S^{k+1} E \rightarrow 0
$$

Then, taking the dual of the sequence and twisting by $L \otimes \operatorname{det} E$, we have the following exact sequences.

$$
\begin{gathered}
0 \rightarrow\left(S^{k+1} E\right)^{\vee} \rightarrow\left(S^{k} E\right)^{\vee} \otimes E^{\vee} \rightarrow\left(S^{k-1} E\right)^{\vee} \otimes(\operatorname{det} E)^{-1} \rightarrow 0 \\
0 \rightarrow\left(S^{k+1} E\right)^{\vee} \otimes(L \otimes \operatorname{det} E) \rightarrow\left(S^{k} E\right)^{\vee} \otimes(E \otimes L) \rightarrow\left(S^{k-1} E\right)^{\vee} \otimes L \rightarrow 0
\end{gathered}
$$

By taking global sections, we get the first exact sequence of the statement. Next, applying $E^{\vee} \cong$ $E \otimes(\operatorname{det} E)^{-1}$ to the last sequence and twisting by $(\operatorname{det} E)^{k-1}$, we have the following exact sequences.

$$
\begin{gathered}
0 \rightarrow S^{k+1} E \otimes(\operatorname{det} E)^{-k} \otimes L \rightarrow S^{k} E \otimes(\operatorname{det} E)^{-(k-1)} \otimes E^{\vee} \otimes L \rightarrow S^{k-1} E \otimes(\operatorname{det} E)^{-(k-1)} \otimes L \rightarrow 0 \\
0 \rightarrow\left(L^{-1} \otimes \operatorname{det} E\right)^{\vee} \otimes S^{k+1} E \rightarrow\left(E \otimes L^{-1}\right)^{\vee} \otimes S^{k} E \rightarrow\left(L^{-1}\right)^{\vee} \otimes S^{k-1} E \rightarrow 0
\end{gathered}
$$

By taking global sections, we obtain the second exact sequence of the statement.

This fact means that if $S^{k+1} E$ is destabilized by a line bundle, then it has implications for the stability of $S^{k} E$. Later, when $E$ has degree 0, using an exact sequence (see Lemma 4.6) which generalizes (3.1), we can deduce that if $S^{k+1} E$ is destabilized by a line subbundle, then, for all $\frac{k+1}{2}<m \leq k, S^{m} E$ is not stable. In the opposite direction, we can show the following.

Proposition 3.2. Let $E$ be a stable vector bundle on $C$ of rank 2 and degree 0 . If $S^{k} E$ is destabilized by a line subbundle $L^{-1} \rightarrow S^{k} E$, then $S^{l} E$ is destabilized by a subbundle $S^{l-k} E \otimes L^{-1} \rightarrow S^{l} E$ for all $l \geq k$.

Proof. If there is a line subbundle $L^{-1} \rightarrow S^{k} E$ of degree 0 , then there exists a $k$-section $D \sim k C_{1}+\mathfrak{b} f$ on $X$ for $L=\mathcal{O}_{C}(\mathfrak{b})$ with projection $\pi=\Pi_{D} \mid: D \rightarrow C$. Thus it gives the exact sequence

$$
0 \rightarrow \mathcal{O}_{X}\left((l-k) C_{1}-\mathfrak{b} f\right) \rightarrow \mathcal{O}_{X}\left(l C_{1}\right) \rightarrow \mathcal{O}_{D}\left(l C_{1}\right) \rightarrow 0
$$

on $X$, and by pushing forward the sequence, we have the following exact sequence on $C$.

$$
\left.0 \rightarrow S^{l-k} E \otimes L^{-1} \rightarrow S^{l} E \rightarrow \pi_{*} \mathcal{O}_{C}\left(l C_{1}\right)\right|_{D} \rightarrow 0
$$

Because $\operatorname{deg}\left(S^{l-k} E \otimes L^{-1}\right)=\operatorname{deg}\left(S^{l} E\right)=0, S^{l-k} E \otimes L^{-1}$ destabilizes $S^{l} E$.

If $S^{3} E$ is destabilized by a line subbundle, then $S^{2} E$ is not stable by Proposition 3.1, so $S^{2} E$ has a destabilizing line subbundle (see Remark (1.1). Conversely, if $S^{2} E$ is destabilized by a line subbundle, then $S^{3} E$ is not stable by Proposition 3.2 , but we do not know whether $S^{3} E$ has a destabilizing subbundle. The following tells when $S^{3} E$ is destabilized by a line subbundle.

Theorem 3.3. Let $E$ be a stable vector bundle on $C$ of rank 2 with trivial determinant. If $S^{3} E$ is destabilized by a line subbundle $L^{-1} \rightarrow S^{3} E$, then $L \in J_{4}(C)$ and $E=\left(\pi_{*} R\right) \otimes L$ for some $R \in \operatorname{Pr}(B / C)$ with $R \in J_{6}(B)$ where $\pi: B \rightarrow C$ is the unramified double covering corresponding to $L^{2} \in J_{2}(C)$. 
Proof. Assume that $S^{3} E$ is destabilized by a line subbundle $L^{-1} \rightarrow S^{3} E$ of $\operatorname{deg} L=0$. Then $S^{2} E$ is destabilized by the subbundle $E \otimes L^{-1} \rightarrow S^{2} E$ by Proposition 3.1. Completing the quotient by comparing the determinants, we have the following exact sequence.

$$
0 \rightarrow E \otimes L^{-1} \rightarrow S^{2} E \rightarrow L^{2} \rightarrow 0
$$

We can observe from Remark 2.3 with the surjection $S^{2} E \rightarrow L^{2}$ that $L^{2} \in J_{2}(C)$. Notice that the dual of the surjection, $L^{-2} \rightarrow\left(S^{2} E\right)^{\vee} \cong S^{2} E$, yields a bisection $B \sim 2 C_{1}+2 \mathfrak{b} f$ on $X=\mathbb{P}_{C}(E)$ for $L=\mathcal{O}_{C}(\mathfrak{b})$. Let $\pi=\left.\Pi\right|_{B}: B \rightarrow C$ be the induced unramified double covering. By pushing forward the exact sequence

$$
0 \rightarrow \mathcal{O}_{X}\left(C_{1}-2 \mathfrak{b} f\right) \rightarrow \mathcal{O}_{X}\left(3 C_{1}\right) \rightarrow \mathcal{O}_{B}\left(3 C_{1}\right) \rightarrow 0
$$

on $X$, we obtain the following exact sequence on $C$.

$$
0 \rightarrow E \otimes L^{-2} \rightarrow S^{3} E \rightarrow \pi_{*} \mathcal{O}_{B}\left(3 C_{1}\right) \rightarrow 0
$$

Since there cannot exist a nonzero morphism $L^{-1} \rightarrow E \otimes L^{-2}$ as $E$ is stable, the morphism $L^{-1} \rightarrow S^{3} E$ induces a nonzero morphism $L^{-1} \rightarrow \pi_{*} \mathcal{O}_{B}\left(3 C_{1}\right)$, and it implies that $\pi_{*} \mathcal{O}_{B}\left(3 C_{1}\right)$ is not stable.

By pushing forward the exact sequence

$$
0 \rightarrow \mathcal{O}_{X}\left(-C_{1}-3 \mathfrak{b} f\right) \rightarrow \mathcal{O}_{X}\left(C_{1}-\mathfrak{b} f\right) \rightarrow \mathcal{O}_{B}\left(C_{1}-\mathfrak{b} f\right) \rightarrow 0
$$

on $X$ to $C$, we get $E \otimes L^{-1}=\Pi_{*} \mathcal{O}_{X}\left(C_{1}-\mathfrak{b} f\right)=\pi_{*} \mathcal{O}_{B}\left(C_{1}-\mathfrak{b} f\right)$. Note that $E \otimes L^{-1}$ is an orthogonal bundle with values in $\mathcal{O}_{C}$ as there is a surjection $S^{2}\left(E \otimes L^{-1}\right) \rightarrow \mathcal{O}_{C}$. Then $\mathcal{O}_{B}\left(C_{1}-\mathfrak{b} f\right) \in \operatorname{Pr}(B / C)$ follows from Mumford's classification.

Because the stability of $\pi_{*} \mathcal{O}_{B}\left(3 C_{1}-3 \mathfrak{b} f\right)=\left(\pi_{*} \mathcal{O}_{B}\left(3 C_{1}\right)\right) \otimes \mathcal{O}_{C}(-3 \mathfrak{b})$ and $\pi_{*} \mathcal{O}_{B}\left(3 C_{1}\right)$ are equivalent, and $\mathcal{O}_{B}\left(3 C_{1}-3 \mathfrak{b} f\right) \in \operatorname{Pr}(B / C)$, we can deduce that $\pi_{*} \mathcal{O}_{B}\left(3 C_{1}-3 \mathfrak{b} f\right)$ splits by Proposition 2.1. Therefore, $\mathcal{O}_{B}\left(3 C_{1}-3 \mathfrak{b} f\right)$ is 2-torsion, and hence $\mathcal{O}_{B}\left(C_{1}-\mathfrak{b} f\right)$ is 6-torsion.

We can verify the converse of the theorem as in the next remark. That is, if $R \in \operatorname{Pr}(B / C) \cap J_{6}(B)$ and $E=\pi_{*} R$, then $S^{3} E$ is destabilized by a line subbundle, but we need to exclude 2-torsion $R$ for $E$ to be stable. For all nontrivial $M \in J_{2}(C)$ and a line bundle $A$ with $A^{2}=M$, recall from Section 2 that the images of $\Phi_{A}: \operatorname{Pr}(B / C) \rightarrow \mathcal{S U}_{C}\left(2, \mathcal{O}_{C}\right)$ are the locus of $E$ with strictly semi-stable $S^{2} E$. Then, $E=\Phi_{A}(R)$ for some $R \in \operatorname{Pr}(B / C) \cap\left(J_{6}(B) \backslash J_{2}(B)\right)$ if and only if $E$ is stable and $S^{3} E$ is destabilized by a line subbundle. Since the number of choices of $M \in J_{2}(C)$ and $R \in J_{6}(B)$ are finite, we can conclude that there are only finitely many such $E$ in $\mathcal{S U}_{C}\left(2, \mathcal{O}_{C}\right)$.

Remark 3.4. Let $\pi: B \rightarrow C$ be an unramified double covering corresponding to $M \in J_{2}(C) \backslash\left\{\mathcal{O}_{C}\right\}$. If $k \geq 3$, then we can find in the same manner that $E=\pi_{*} R$ is stable and $S^{k} E$ is destabilized by a line subbundle for $R \in \operatorname{Pr}(B / C) \cap\left(J_{2 k}(B) \backslash J_{2}(B)\right)$. Let $X=\mathbb{P}_{C}(E)$ be the ruled surface $\Pi: \mathbb{P}_{C}(E) \rightarrow C$ and $C_{1}$ be a unisecant divisor on $X$ such that $\Pi_{*} \mathcal{O}_{X}\left(C_{1}\right)=E$. As $S^{2} E$ is destabilized by $\mathcal{O}_{C}, B$ realizes as a bisection $B \sim 2 C_{1}$ on $X$, and then $E=\pi_{*} \mathcal{O}_{B}\left(C_{1}\right)$. By pushing forward the exact sequence

$$
0 \rightarrow \mathcal{O}_{X}\left((k-2) C_{1}\right) \rightarrow \mathcal{O}_{X}\left(k C_{1}\right) \rightarrow \mathcal{O}_{B}\left(k C_{1}\right) \rightarrow 0
$$

on $X$, we obtain the following exact sequence on $C$.

$$
0 \rightarrow S^{k-2} E \rightarrow S^{k} E \rightarrow \pi_{*} \mathcal{O}_{B}\left(k C_{1}\right) \rightarrow 0
$$

Notice that $E$ is orthogonal with values in $\mathcal{O}_{C}$. Thus $\mathcal{O}_{B}\left(C_{1}\right) \in \operatorname{Pr}(B / C)$, and so $\mathcal{O}_{B}\left(k C_{1}\right) \in \operatorname{Pr}(B / C)$. Hence $\mathcal{O}_{B}\left(k C_{1}\right) \in J_{2}(B)$ is equivalent to saying that $\pi_{*} \mathcal{O}_{B}\left(k C_{1}\right)$ splits into the direct sum of line bundles by Proposition 2.1 Therefore, $S^{k} E$ is destabilized by a line (sub)bundle when $\mathcal{O}_{B}\left(C_{1}\right)$ is $2 k$-torsion. 
3.2. Destabilized by Rank 2 but not by Rank 1. In Proposition 3.2 we observe that if $S^{2} E$ is strictly semi-stable, then $S^{3} E$ is destabilized by a subbundle of rank 2 . The converse does not hold as there is an example where $S^{2} E$ is stable but $S^{4} E$ is destabilized by a line subbundle. By Proposition 3.1. if $S^{4} E$ is destabilized by a line subbundle, then $S^{3} E$ is destabilized by a subbundle of rank 2 .

Example 3.5. Let $\eta: C^{\prime} \rightarrow C$ be an unramified cyclic triple covering corresponding to $L \in J_{3}(C) \backslash\left\{\mathcal{O}_{C}\right\}$. Then we have $\eta_{*} \mathcal{O}_{C^{\prime}}=\mathcal{O}_{C} \oplus L^{-1} \oplus L^{-2}$. For $M \in J_{2}\left(C^{\prime}\right), V=\eta_{*} M$ becomes an orthogonal bundle on $C$ of rank 3. Indeed, the surjection

$$
\eta^{*} S^{2} V=S^{2}\left(\eta^{*} V\right)=S^{2}\left(\eta^{*}\left(\eta_{*} M\right)\right) \rightarrow M^{2}=\mathcal{O}_{C^{\prime}}
$$

induces a nonzero morphism $S^{2} V \rightarrow \eta_{*} \mathcal{O}_{C^{\prime}}=\mathcal{O}_{C} \oplus L^{-1} \oplus L^{-2}$, and it implies that $V$ is orthogonal because one of $S^{2} V \rightarrow L^{-k}$ is nonzero which is necessarily surjective due to the semi-stability of $S^{2} V$. In fact, there are surjections $S^{2} V \rightarrow L^{-k}$ for all $k(\bmod 3)$; if there is a surjection $S^{2} V \rightarrow L^{-k}$ for one $k$, then the isomorphism $V \otimes L=\eta_{*} M \otimes L \cong \eta_{*}\left(M \otimes \eta^{*} L\right) \cong \eta_{*} M=V$ induces another surjection $S^{2} V \rightarrow L^{-k+1}$ after twisting the surjection $S^{2} V \otimes L^{2} \cong S^{2}(V \otimes L) \cong S^{2} V \rightarrow L^{-k}$ by $L$.

According to Mumford's classification [16] of orthogonal bundles of rank $3, V \cong A^{-1} \otimes S^{2} E$ for some vector bundle $E$ of rank 2 and line bundle $A$ satisfying $A^{2}=(\operatorname{det} E)^{2}$. We will first check that $S^{2} E$ is stable if and only if $M \notin \eta^{*} J^{0}(C)$, and then show that $S^{4} E$ has a destabilizing quotient line bundle.

If $\eta_{*} M$ is destabilized by a line subbundle $R^{-1} \rightarrow \eta_{*} M$ of degree 0 , then there is a nonzero morphism $\eta^{*} R^{-1} \rightarrow M$. Since both $M$ and $\eta^{*} R^{-1}$ have degree 0 , it is possible if and only if $M=\eta^{*} R^{-1} \in \eta^{*} J^{0}(C)$.

Next, to show that $S^{4} E$ is destabilized by the quotient line bundles $S^{4} E \rightarrow A^{2} \otimes L^{ \pm 1}$, we will use the following exact sequence obtained by completing the kernel of the natural surjection $S^{2}\left(S^{2} E\right) \rightarrow S^{4} E$ after comparing the determinants.

$$
0 \rightarrow(\operatorname{det} E)^{2} \rightarrow S^{2}\left(S^{2} E\right) \rightarrow S^{4} E \rightarrow 0
$$

Twisting the sequence by $A^{-2} \cong(\operatorname{det} E)^{2}$, we have the following exact sequence.

$$
0 \rightarrow \mathcal{O}_{C} \rightarrow S^{2}\left(A^{-1} \otimes S^{2} E\right) \rightarrow S^{4} E \otimes A^{-2} \rightarrow 0
$$

Because there exist the surjections $S^{2} V \rightarrow L^{ \pm 1}$ and $L \neq \mathcal{O}_{C}$, the above sequence with $V \cong A^{-1} \otimes S^{2} E$ induces surjections $S^{4} E \otimes A^{-2} \rightarrow L^{ \pm 1}$. That is, $S^{4} E$ has destabilizing quotient bundles $A^{2} \otimes L^{ \pm 1}$.

Note that the number of $E$ given by the above construction is finite since there are only finitely many $m$-torsion line bundles for each $m$ and vector bundles $E$ with $\operatorname{det} E=\operatorname{det} F$ which satisfy $S^{2} E=S^{2} F$ for a fixed vector bundle $F$ of rank 2 with even degree when $S^{2} F$ is stable. As the first fact is well-known, we give a proof for the latter fact.

Proposition 3.6. Let $E, F$ be vector bundles on $C$ of rank 2 with the same determinant of even degree. If $S^{2} E, S^{2} F$ are stable and $S^{2} E \cong S^{2} F$, then $E \cong F \otimes M$ for some 2-torsion line bundle $M$.

Proof. We may give a proof under the assumption that $\operatorname{det} E=\operatorname{det} F=\mathcal{O}_{C}$. Notice that $E, F$ are stable because $S^{2} E, S^{2} F$ are stable. Due to $E \otimes E=\mathcal{O}_{C} \oplus S^{2} E, E^{\vee} \cong E$, and the same facts for $F$, we have

$$
\begin{aligned}
\operatorname{dim} \operatorname{Hom}(E \otimes F, E \otimes F) & =h^{0}\left(E^{\vee} \otimes F^{\vee} \otimes E \otimes F\right)=\operatorname{dim} \operatorname{Hom}(E \otimes E, F \otimes F) \\
& \geq \operatorname{dim} \operatorname{Hom}\left(\mathcal{O}_{C}, \mathcal{O}_{C}\right)+\operatorname{dim} \operatorname{Hom}\left(S^{2} E, S^{2} F\right)=2 .
\end{aligned}
$$

Thus $E \otimes F$ is not simple, so not stable, hence there exists a subbundle $V \rightarrow E \otimes F$ of rank $r \leq 2$ and degree 0 by Remark 1.1. If $r=1$, then it induces an isomorphism $E \cong E^{\vee} \cong F \otimes V^{-1}$ as $E$ and $F$ are stable. After comparing the determinants, we can check that $M^{2}=\operatorname{det}\left(F \otimes V^{-1}\right)=\operatorname{det} E=\mathcal{O}_{C}$ for $M=V^{-1}$ which gives an isomorphism $E \cong F \otimes M$. 
Otherwise, if there is no destabilizing line subbundle of $E \otimes F$ so that $r=2$, then we can derive a contradiction as follows. Suppose that there is an exact sequence

$$
0 \rightarrow V \rightarrow E \otimes F \rightarrow W \rightarrow 0
$$

for some vector bundles $V$ and $W$ of rank 2 and degree 0 . If $W$ is not stable so there exists a destabilizing quotient line bundle $W \rightarrow L$, then it gives a destabilizing line subbundle $L^{-1} \rightarrow W^{\vee} \rightarrow(E \otimes F)^{\vee} \cong E \otimes F$. If $V$ is not stable, then we have the same contradiction. So we assume that both $V$ and $W$ are stable. Then, using the filtration

$$
S^{2}(E \otimes F)=G_{0} \supseteq G_{1} \supseteq G_{2} \supseteq G_{3}=0
$$

satisfying $G_{0} / G_{1}=S^{2} W, G_{1} / G_{2}=V \otimes W$, and $G_{2} / G_{3}=S^{2} V$, we obtain that

$$
h^{0}\left(S^{2}(E \otimes F)\right) \leq h^{0}\left(S^{2} V\right)+h^{0}\left(S^{2} W\right)+h^{0}(V \otimes W) .
$$

On the other hand, by the isomorphism

$$
S^{2}(E \otimes F) \cong(\operatorname{det} E \otimes \operatorname{det} F) \oplus\left(S^{2} E \otimes S^{2} F\right)=\mathcal{O}_{C} \oplus\left(S^{2} E \otimes S^{2} F\right),
$$

we have $h^{0}\left(S^{2}(E \otimes F)\right)=h^{0}\left(\mathcal{O}_{C}\right)+\operatorname{dim} \operatorname{Hom}\left(\left(S^{2} E\right)^{\vee}, S^{2} F\right)=1+\operatorname{dim} \operatorname{Hom}\left(S^{2} E, S^{2} F\right)=2$, and therefore,

$$
h^{0}\left(S^{2} V\right)+h^{0}\left(S^{2} W\right)+h^{0}(V \otimes W) \geq 2 .
$$

We will first show that $h^{0}\left(S^{2} V\right)=h^{0}\left(S^{2} W\right)=1$. That is, $V$ and $W$ are orthogonal with values in $\mathcal{O}_{C}$. Since $V \otimes V=\operatorname{det} V \oplus S^{2} V$ and $V$ is stable, $h^{0}\left(S^{2} V\right) \leq h^{0}(V \otimes V)=\operatorname{dim} \operatorname{Hom}\left(V^{\vee}, V\right) \leq 1$. For the same reason, $h^{0}\left(S^{2} W\right) \leq 1$. Thus it suffices to treat the case $h^{0}(V \otimes W) \neq 0$. Because both $V$ and $W$ are stable, $h^{0}(V \otimes W)=\operatorname{dim} \operatorname{Hom}\left(V^{\vee}, W\right) \leq 1$, and the equality holds if and only if $W \cong V^{\vee}$. Hence if $h^{0}(V \otimes W) \neq 0$, then $h^{0}(V \otimes W)=1$, so we have either $h^{0}\left(S^{2} V\right)=1$ or $h^{0}\left(S^{2} W\right)=1$ from $h^{0}\left(S^{2} V\right)+h^{0}\left(S^{2} W\right) \geq 1$. Without loss of generality, we may assume that $h^{0}\left(S^{2} V\right)=1$, equivalently, there is a symmetric isomorphism $V^{\vee} \cong V$. Then $W \cong V^{\vee} \cong V$ is true due to $h^{0}(V \otimes W) \neq 0$. Thus we get $S^{2} W \cong S^{2} V$ as well, and so $h^{0}\left(S^{2} W\right)=h^{0}\left(S^{2} V\right)=1$.

Now suppose that $V$ and $W$ are orthogonal with values in $\mathcal{O}_{C}$. By Remark 2.4, we know that det $V$ and $\operatorname{det} W$ are nontrivial 2-torsion. From $\operatorname{det} V \otimes \operatorname{det} W=\operatorname{det}(E \otimes F)=\mathcal{O}_{C}$, we also have $\operatorname{det} V=\operatorname{det} W$, and denote them by $N=\operatorname{det} V=\operatorname{det} W \in J_{2}(C)$. Then, from $V \otimes N \cong V^{\vee} \cong V$ and $W \otimes N \cong W^{\vee} \cong W$, we obtain that $(E \otimes F) \otimes N \cong E \otimes F$ since $E \otimes F$ is an extension of $W$ by $V$. Thus

$$
\begin{aligned}
(E \otimes F) \otimes(E \otimes F) & \cong(E \otimes F) \otimes((E \otimes F) \otimes N)=((E \otimes E) \otimes(F \otimes F)) \otimes N \\
& =\left(\left(\mathcal{O}_{C} \oplus S^{2} E\right) \otimes\left(\mathcal{O}_{C} \oplus S^{2} F\right)\right) \otimes N=\left(\mathcal{O}_{C} \oplus S^{2} E \oplus S^{2} F \oplus\left(S^{2} E \otimes S^{2} F\right)\right) \otimes N \\
& =N \oplus\left(S^{2} E \otimes N\right) \oplus\left(S^{2} F \otimes N\right) \oplus\left(S^{2} E \otimes S^{2} F \otimes N\right)
\end{aligned}
$$

implies that $h^{0}(N)+h^{0}\left(S^{2} E \otimes N\right)+h^{0}\left(S^{2} F \otimes N\right)+h^{0}\left(S^{2} E \otimes S^{2} F \otimes N\right)=\operatorname{dim} \operatorname{Hom}(E \otimes F, E \otimes F)=2$.

Because $S^{2} E \cong S^{2} F$ are stable, $h^{0}\left(S^{2} E \otimes N\right)=h^{0}\left(S^{2} F \otimes N\right)=0$. Moreover, due to their stability,

$$
h^{0}\left(S^{2} E \otimes S^{2} F \otimes N\right)=\operatorname{dim} \operatorname{Hom}\left(\left(S^{2} E\right)^{\vee}, S^{2} F \otimes N\right)=\operatorname{dim} \operatorname{Hom}\left(S^{2} E, S^{2} F \otimes N\right) \leq 1 .
$$

Thus it follows that $h^{0}(N)=1$ which is equivalent to saying that $\operatorname{det} V=N=\mathcal{O}_{C}$. Then it contradicts the inequality $h^{0}(\operatorname{det} V)+h^{0}\left(S^{2} V\right)=h^{0}(V \otimes V)=\operatorname{dim} \operatorname{Hom}\left(V^{\vee}, V\right) \leq 1$ satisfied by the stability of $V$. Therefore, there must be a line subbundle $M^{-1} \rightarrow E \otimes F$ yielding an isomorphism $E \cong E^{\vee} \cong F \otimes M$.

The remaining part of this subsection is devoted to showing that the vector bundles constructed in Example 3.5 are the only vector bundles $E$ where $S^{2} E$ is stable but $S^{3} E$ is not stable. 
Lemma 3.7. Let $E$ be a vector bundle on $C$ of rank 2 and degree 0 . Assume that $S^{k} E$ is destabilized by a line subbundle so there exists a $k$-section $D$ of zero self-intersection on the ruled surface $X=\mathbb{P}_{C}(E)$. Then $D$ is irreducible and reduced if $S^{m} E$ is not destabilized by a line subbundle for any $m \leq \frac{k}{2}$, or equivalently, if there is no $m$-section of zero self-intersection on $X$ for any $m \leq \frac{k}{2}$ (see Remark 2.6).

Proof. We fix a unisecant divisor $C_{1}$ on $X$ satisfying $C_{1}{ }^{2}=0$. Then necessarily $D \equiv k C_{1}$. If $D$ is neither irreducible nor reduced, then $D=D_{1} \cup D_{2}$ for some effective divisors $D_{1} \equiv m C_{1}+b f$ and $D_{2} \equiv n C_{1}+d f$ with $m+n=k$ for $m, n>0$ and $b+d=0$ for $b, d \geq 0$. So we have $b=d=0$ and both $D_{1}$ and $D_{2}$ have zero self-intersection on $X$. Thus it yields a contradiction as one of $m \leq \frac{k}{2}$ or $n \leq \frac{k}{2}$ must hold.

Lemma 3.8. Let $E$ be a vector bundle on $C$ of rank 2 and degree 0 and $X=\mathbb{P}_{C}(E)$ be the ruled surface $\Pi: \mathbb{P}_{C}(E) \rightarrow C$ with a unisecant divisor $C_{1}$ on $X$ satisfying $\Pi_{*} \mathcal{O}_{X}\left(C_{1}\right) \cong E$. Assume that $k \geq 2$ and $S^{m} E$ is not destabilized by a line subbundle for any $m \leq k$. If there exists an injection $V \rightarrow S^{k} E$ for some stable vector bundle $V$ on $C$ of rank 2 and degree 0 , then it induces a surjection $\Pi^{*} V \rightarrow \mathcal{O}_{X}\left(k C_{1}\right)$ on $X$.

Proof. There is a nonzero morphism $\gamma: \Pi^{*} V \rightarrow \mathcal{O}_{X}\left(k C_{1}\right)$ from the adjoint correspondence

$$
\operatorname{Hom}\left(\Pi^{*} V, \mathcal{O}_{X}\left(k C_{1}\right)\right) \cong \operatorname{Hom}\left(V, \Pi_{*} \mathcal{O}_{X}\left(k C_{1}\right)\right)=\operatorname{Hom}\left(V, S^{k} E\right) .
$$

Since $\operatorname{im} \gamma$ is a torsion-free sheaf on $X$ of rank 1 , we can write $\operatorname{im} \gamma=\mathcal{O}_{X}\left(m C_{1}+\mathfrak{d} f\right) \otimes \mathcal{I}_{Z}$ for some integer $m, \mathfrak{d} \in \operatorname{Pic}(C)$, and 0 -dimensional subscheme $Z$ of $X$, which may be empty. As the injection $\operatorname{im} \gamma \rightarrow \mathcal{O}_{X}\left(k C_{1}\right)$ induces a nonzero morphism $\mathcal{O}_{X}\left(m C_{1}+\mathfrak{d} f\right) \rightarrow \mathcal{O}_{X}\left(k C_{1}\right)$ between the reflexive hulls, $m \leq k$ and $\operatorname{deg} \mathfrak{d} \leq 0$ must hold.

Let $P \in C$ be arbitrary and $P f=\Pi^{-1}(P)$ denote the $\mathbb{P}^{1}$-fiber of $\Pi$ over $P$. Since there is a trivialization $\left.V\right|_{U} \cong \mathcal{O}_{U} \oplus \mathcal{O}_{U}$ in a neighborhood $U \subseteq C$ of $P \in C$, we get $\left.\Pi^{*} V\right|_{P f} \cong \mathcal{O}_{\mathbb{P}^{1}} \oplus \mathcal{O}_{\mathbb{P}^{1}}$. Then, by restricting the surjection $\Pi^{*} V \rightarrow \operatorname{im} \gamma$ onto $P f$, we obtain a surjection $\mathcal{O}_{\mathbb{P}^{1}} \oplus \mathcal{O}_{\mathbb{P}^{1}} \rightarrow \mathcal{O}_{\mathbb{P}^{1}}(m)$. Since $\mathcal{O}_{\mathbb{P}^{1}}(m)$ cannot be generated by global sections when $m<0$, we get $m \geq 0$.

On the other hand, by restricting the surjection $\Pi^{*} V \rightarrow \operatorname{im} \gamma$ onto an $l$-section $D \sim l C_{1}+\mathfrak{c} f$ for some $\mathfrak{c} \in \operatorname{Pic}(C)$ of $\operatorname{deg} \mathfrak{c}=c$, we have a surjection $\left.\left.\Pi^{*} V\right|_{D} \rightarrow \mathcal{O}_{X}\left(m C_{1}+\mathfrak{d} f\right) \otimes \mathcal{I}_{Z}\right|_{D}$. Because $\pi=\left.\Pi\right|_{D}: D \rightarrow C$ is finite, $\left.\Pi^{*} V\right|_{D}=\pi^{*} V$ is a semi-stable vector bundle on $D$ of degree 0 [14, II: p. 61]. Thus

$$
0 \leq\left.\operatorname{deg} \mathcal{O}_{X}\left(m C_{1}+\mathfrak{d} f\right) \otimes \mathcal{I}_{Z}\right|_{D} \leq\left(m C_{1}+\mathfrak{d} f\right) \cdot\left(l C_{1}+\mathfrak{c} f\right)=m c+l \operatorname{deg} \mathfrak{d} .
$$

Since the $\mathbb{R}$-ray generated by $\left[C_{1}\right]$ lies on the boundary of the cone of ample divisors in $N^{1}(X)$, we can choose a smooth curve $D \equiv l C_{1}+c f$ with $c / l>0$ being arbitrary small by taking sufficiently large $l \gg 0$. So $\operatorname{deg} \mathfrak{d}$ is necessarily 0 .

Let $L=\mathcal{O}_{C}(\mathfrak{b})=\operatorname{det} V$ and consider the exact sequence

$$
0 \rightarrow \mathcal{O}_{X}\left(n C_{1}+\mathfrak{e} f\right) \otimes \mathcal{I}_{W} \rightarrow \Pi^{*} V \rightarrow \mathcal{O}_{X}\left(m C_{1}+\mathfrak{d} f\right) \otimes \mathcal{I}_{Z} \rightarrow 0
$$

on $X$ for some integer $n, \mathfrak{e} \in \operatorname{Pic}(C)$, and 0-dimensional subscheme $W$ of $X$, which is possibly empty. Applying Whitney's formula for coherent sheaves to the sequence, we get

$$
c_{1}\left(\Pi^{*} V\right)=\left(m C_{1}+\mathfrak{d} f\right)+\left(n C_{1}+\mathfrak{e} f\right) \text { and } c_{2}\left(\Pi^{*} V\right)=\left(m C_{1}+\mathfrak{d} f\right) \cdot\left(n C_{1}+\mathfrak{e} f\right)+\operatorname{len}(Z)+\operatorname{len}(W) .
$$

Because $c_{1}\left(\Pi^{*} V\right)=\mathfrak{b} f, c_{2}\left(\Pi^{*} V\right)=0$, and $C_{1}{ }^{2}=0$, we obtain $n=-m, \mathfrak{e}=\mathfrak{b}-\mathfrak{d}$, and $Z=W=\emptyset$. Then, from the inclusion $\mathcal{O}_{X}\left(m C_{1}+\mathfrak{d} f\right) \rightarrow \mathcal{O}_{X}\left(k C_{1}\right)$, we get a global section of $\mathcal{O}_{X}\left((k-m) C_{1}-\mathfrak{d} f\right)$, which gives a $(k-m)$-section of zero self-intersection on $X$ if $m<k$, so it contradicts the assumption. Therefore, $m=k$ and hence $\mathfrak{d}=0$, which together imply that $\gamma$ is surjective. 
Proposition 3.9. Let $E$ be a vector bundle on $C$ of rank 2 with trivial determinant. Assume that $S^{2} E$ is stable and $S^{3} E$ is not destabilized by a line subbundle. If $S^{3} E$ is destabilized by a subbundle $V \rightarrow S^{3} E$ of rank 2, then $L=\operatorname{det} V$ is nontrivial 3-torsion, and $S^{3} E$ has two distinct destabilizing subbundles $E \otimes L^{\mp 1} \rightarrow S^{3} E$.

Proof. Assume that there exists a nonzero morphism $V \rightarrow S^{3} E$ for some stable vector bundle $V$ on $C$ of rank 2 with $\operatorname{det} V=L$ of $\operatorname{deg} L=0$. Then, by Lemma 3.8, the induced morphism $\Pi^{*} V \rightarrow \mathcal{O}_{X}\left(3 C_{1}\right)$ must be surjective where $X=\mathbb{P}_{C}(E)$ is the ruled surface $\Pi: \mathbb{P}_{C}(E) \rightarrow C$ and $C_{1}$ is a unisecant divisor on $X$ such that $E=\Pi_{*} \mathcal{O}_{X}\left(C_{1}\right)$. Then we have the following exact sequence on $X$ by completing the kernel after comparing the determinants.

$$
0 \rightarrow \mathcal{O}\left(-3 C_{1}\right) \otimes \Pi^{*} L \rightarrow \Pi^{*} V \rightarrow \mathcal{O}_{X}\left(3 C_{1}\right) \rightarrow 0
$$

By pushing forward the above sequence, we get the exact sequence

$$
0 \rightarrow V \rightarrow S^{3} E \rightarrow E^{\vee} \otimes L \rightarrow 0
$$

on $C$, and it gives that $L^{3}=\mathcal{O}_{C}$ by comparing the determinants. Moreover, by taking the dual of the sequence, we obtain an injection $E \otimes L^{-1} \rightarrow\left(S^{3} E\right)^{\vee} \cong S^{3} E$.

Assume that $L$ is nontrivial. Due to Lemma 3.8 with respect to $E \otimes L^{-1} \rightarrow S^{3} E$, we can say that there exists the following exact sequence on $X$.

$$
0 \rightarrow \mathcal{O}_{X}\left(-3 C_{1}\right) \otimes \Pi^{*} L^{-2} \rightarrow \Pi^{*}\left(E \otimes L^{-1}\right) \rightarrow \mathcal{O}_{X}\left(3 C_{1}\right) \rightarrow 0
$$

By pushing forward the exact sequence after twisting by $\mathcal{O}_{X}\left(-C_{1}\right)$, we have the exact sequence

$$
0 \rightarrow S^{2} E \rightarrow S^{2} E \otimes L^{-2} \rightarrow 0
$$

on $C$ due to $\left(S^{2} E\right)^{\vee} \cong S^{2}\left(E^{\vee}\right) \cong S^{2} E$. Thus $S^{2} E \cong S^{2} E \otimes L$. Twisting both sides by $L$, we also obtain that $S^{2} E \otimes L \cong S^{2} E \otimes L^{2}$. That is, $S^{2} E \otimes L^{-1} \cong S^{2} E \cong S^{2} E \otimes L$. As shown in Example 3.5 there is the following exact sequence.

$$
0 \rightarrow \mathcal{O}_{C} \rightarrow S^{2}\left(S^{2} E\right) \rightarrow S^{4} E \rightarrow 0
$$

Notice that $H^{0}\left(S^{2}\left(S^{2} E\right)\right) \neq 0$. Twisting the sequence by $L^{ \pm 1}$, we get the following exact sequence.

$$
0 \rightarrow L^{ \pm 1} \rightarrow S^{2}\left(S^{2} E\right) \rightarrow S^{4} E \otimes L^{ \pm 1} \rightarrow 0
$$

Since $L \neq \mathcal{O}_{C}, H^{0}\left(S^{2}\left(S^{2} E\right)\right) \neq 0$ implies that $H^{0}\left(S^{4} E \otimes L^{ \pm 1}\right) \neq 0$. So there are nonzero morphisms $L^{\mp 1} \rightarrow S^{4} E$, which respectively induce destabilizations $E \otimes L^{\mp 1} \rightarrow S^{3} E$ by Proposition 3.1,

Now it remains to eliminate the case $L=\mathcal{O}_{C}$. Suppose that there is an injection $E \rightarrow S^{3} E$. Applying Proposition 3.1 we can see that there exist a 4 -section $D \sim 4 C_{1}$ and the following exact sequence on $X$.

$$
0 \rightarrow \mathcal{O}_{X}\left(-8 C_{1}\right) \rightarrow \mathcal{O}_{X}\left(-4 C_{1}\right) \rightarrow \mathcal{O}_{D}\left(-4 C_{1}\right) \rightarrow 0
$$

By pushing forward the sequence to $C$, we obtain an injection $H^{0}\left(\pi_{*} \mathcal{O}_{D}\left(-4 C_{1}\right)\right) \rightarrow H^{0}\left(S^{6} E\right)$ after taking global sections where $\pi: D \rightarrow C$ is the induced unramified 4-covering. As $S^{2} E$ is assumed to be stable so that $E$ is stable as well, $D$ is irreducible and reduced by Lemma 3.7. Due to Proposition 2.7. $\mathcal{O}_{D}\left(2 C_{1}\right)=\mathcal{O}_{D}$, and thus $H^{0}\left(S^{6} E\right) \neq 0$ follows from $H^{0}\left(\pi_{*} \mathcal{O}_{D}\left(-4 C_{1}\right)\right)=H^{0}\left(\pi_{*} \mathcal{O}_{D}\right)=H^{0}\left(\mathcal{O}_{D}\right) \neq 0$.

Let $B \sim 6 C_{1}$ be the 6 -section on $X$ corresponding to a nonzero global section of $S^{6} E$, and we denote the induced 6-covering by $\xi=\left.\Pi\right|_{B}: B \rightarrow C$. Suppose that $B$ is irreducible and reduced. Then, from Remark 2.6, $B$ is smooth. By pushing forward the exact sequence

$$
0 \rightarrow \mathcal{O}_{X}\left(-6 C_{1}\right) \rightarrow \mathcal{O}_{X} \rightarrow \mathcal{O}_{B} \rightarrow 0
$$


on $X$ to $C$, we can see that $\xi_{*} \mathcal{O}_{B}=\mathcal{O}_{C} \oplus S^{4} E$ as there is a splitting of the natural inclusion $\mathcal{O}_{C} \rightarrow \xi_{*} \mathcal{O}_{B}$ [14 I: p. 248]. Because $H^{0}\left(S^{4} E\right) \neq 0$, we get $h^{0}\left(\mathcal{O}_{B}\right)=h^{0}\left(\xi_{*} \mathcal{O}_{B}\right) \geq 2$, contradicting the hypothesis on $B$. Therefore, $B$ is neither irreducible nor reduced, and so by Lemma 3.7, we have a contradiction to the assumption that none of $E, S^{2} E$, and $S^{3} E$ are destabilized by a line subbundle.

We continue to classify vector bundles $E$ of rank 2 with trivial determinant where $S^{2} E$ is stable but there exist subbundles $E \otimes L^{\mp 1} \rightarrow S^{3} E$ for some nontrivial 3-torsion line bundle $L$.

Proposition 3.10. Let $E$ be a vector bundle on $C$ of rank 2 with trivial determinant. Assume that $S^{2} E$ is stable and $S^{3} E$ has destabilizing subbundles $E \otimes L^{\mp 1} \rightarrow S^{3} E$ for some $L \in J_{3}(C) \backslash\left\{\mathcal{O}_{C}\right\}$. Then there exists an irreducible and reduced 6-section $B$ on $X=\mathbb{P}_{C}(E)$ and $\xi^{*} L=\mathcal{O}_{B}$ for the induced 6-covering $\xi: B \rightarrow C$.

Proof. Using Lemma 3.8 with respect to the inclusions $E \otimes L^{\mp 1} \rightarrow S^{3} E$, we can further assert that there exist two exact sequences

$$
0 \rightarrow \mathcal{O}_{X}\left(-3 C_{1}\right) \otimes \Pi^{*} L^{\mp 2} \rightarrow \Pi^{*}\left(E \otimes L^{\mp 1}\right) \rightarrow \mathcal{O}_{X}\left(3 C_{1}\right) \rightarrow 0
$$

on $X$. After twisting by $\Pi^{*} L^{ \pm 1}=\Pi^{*} \mathcal{O}_{C}( \pm \mathfrak{b})=\mathcal{O}_{X}( \pm \mathfrak{b} f)$, the sequences are in other way written by

$$
0 \rightarrow \mathcal{O}_{X}\left(-3 C_{1} \mp \mathfrak{b} f\right) \rightarrow \Pi^{*} E \rightarrow \mathcal{O}_{X}\left(3 C_{1} \pm \mathfrak{b} f\right) \rightarrow 0 .
$$

Since $L$ is nontrivial 3-torsion, $\mathcal{O}_{X}\left(3 C_{1}+\mathfrak{a} f\right) \neq \mathcal{O}_{X}\left(3 C_{1}-\mathfrak{a} f\right)$. So, by adjoining

$$
0 \rightarrow \mathcal{O}_{X}\left(-3 C_{1}+\mathfrak{b} f\right) \rightarrow \Pi^{*} E \text { and } \Pi^{*} E \rightarrow \mathcal{O}_{X}\left(3 C_{1}+\mathfrak{b} f\right) \rightarrow 0,
$$

we get a nonzero morphism $\mathcal{O}_{X}\left(-3 C_{1}+\mathfrak{b} f\right) \rightarrow \mathcal{O}_{X}\left(3 C_{1}+\mathfrak{b} f\right)$ which yields a global section of $\mathcal{O}_{X}\left(6 C_{1}\right)$. Thus if $S^{3} E$ has destabilizing subbundles $E \otimes L^{\mp 1} \rightarrow S^{3} E$ for some $L \in J_{3}(C) \backslash\left\{\mathcal{O}_{C}\right\}$, then there exists a 6 -section $B \sim 6 C_{1}$ on $X$. Furthermore, by Lemma 3.7 $B$ is irreducible and reduced as $S^{2} E$ is stable.

Let $\xi=\left.\Pi\right|_{B}: B \rightarrow C$ be the induced 6-covering. By restricting the three surjections

$$
\Pi^{*} E \rightarrow \mathcal{O}_{X}\left(3 C_{1}+\mathfrak{b} f\right), \quad \Pi^{*} E \rightarrow \mathcal{O}_{X}\left(3 C_{1}-\mathfrak{b} f\right), \text { and } \Pi^{*} E \rightarrow \mathcal{O}_{X}\left(C_{1}\right)
$$

on $X$ to $B$, we have the following three quotient line bundles on $B$ of degree 0 .

$$
\xi^{*} E \rightarrow \mathcal{O}_{B}\left(3 C_{1}+\mathfrak{b} f\right), \quad \xi^{*} E \rightarrow \mathcal{O}_{B}\left(3 C_{1}-\mathfrak{b} f\right), \text { and } \xi^{*} E \rightarrow \mathcal{O}_{B}\left(C_{1}\right)
$$

As $B \sim 6 C_{1}$ is effective, by pushing forward the exact sequences

$$
0 \rightarrow \mathcal{O}_{X}\left(-4 C_{1} \pm \mathfrak{b} f\right) \rightarrow \mathcal{O}_{X}\left(2 C_{1} \pm \mathfrak{b} f\right) \rightarrow \mathcal{O}_{B}\left(2 C_{1} \pm \mathfrak{a} f\right) \rightarrow 0
$$

on $X$, we have the exact sequences

$$
0 \rightarrow S^{2} E \rightarrow \xi_{*} \mathcal{O}_{B}\left(2 C_{1} \pm \mathfrak{b} f\right) \rightarrow S^{2} E \rightarrow 0
$$

on $C$ because $\left(S^{2} E\right)^{\vee} \cong S^{2} E$ and $S^{2} E \cong S^{2} E \otimes L^{ \pm 1}$ from (3.2) in the proof of Proposition 3.9.

Now suppose that $\xi^{*} L=\mathcal{O}_{B}(\mathfrak{b} f)$ is nontrivial. Because $\xi^{*} E$ is a semi-stable vector bundle on $B$ of rank 2 and degree 0 , it has at most two distinct isomorphic types of quotient line bundles of degree 0 . Since the first and second quotients in (3.3) are distinct, the third quotient must be equal to one of the others. Thus we get either

$$
\mathcal{O}_{B}\left(2 C_{1}+\mathfrak{b} f\right) \cong \mathcal{O}_{B} \quad \text { or } \quad \mathcal{O}_{B}\left(2 C_{1}-\mathfrak{b} f\right) \cong \mathcal{O}_{B}
$$

So, when $\mathcal{O}_{B}(\mathfrak{b} f) \neq \mathcal{O}_{B}, H^{0}\left(S^{2} E\right) \neq 0$ follows from one of (3.4) together with $h^{0}\left(\xi_{*} \mathcal{O}_{B}\right)=h^{0}\left(\mathcal{O}_{B}\right)=1$, and it gives rise to the strict semi-stability of $S^{2} E$, contradicting the assumption. Thus $\xi^{*} L=\mathcal{O}_{B}$. 
Theorem 3.11. Let $E$ be a vector bundle on $C$ of rank 2 with trivial determinant. Assume that $S^{2} E$ is stable but $S^{3} E$ is not destabilized by a line subbundle. If $S^{3} E$ is destabilized by a subbundle of rank 2, then $S^{2} E=\eta_{*} M$ for some nontrivial unramified cyclic covering $\eta: C^{\prime} \rightarrow C$ of degree 3 and 2-torsion line bundle $M$ on $C^{\prime}$ which is not contained in $\eta^{*} J_{0}(C)$.

Proof. Let $X=\mathbb{P}_{C}(E)$ be the ruled surface $\Pi: \mathbb{P}_{C}(E) \rightarrow C$ and $C_{1}$ be a unisecant divisor on $X$ such that $\Pi_{*} \mathcal{O}_{X}\left(C_{1}\right)=E$. Under the same assumption, we have seen in Proposition 3.9 and 3.10 that

(1) there exists an irreducible and reduced 6 -section $B \sim 6 C_{1}$ on $X$ with projection $\xi=\left.\Pi\right|_{B}: B \rightarrow C$,

(2) there exists a nontrivial 3-torsion line bundle $L=\mathcal{O}_{C}(\mathfrak{b})$ on $C$ and $\xi^{*} L$ is trivial,

(3) from (3.3), $\xi^{*} E$ has quotient line bundles $\mathcal{O}_{B}\left(C_{1}\right)$ and $\mathcal{O}_{B}\left(3 C_{1}\right)$ of degree 0 , and

(4) from (3.4), there is the following exact sequence on $C$.

$$
0 \rightarrow S^{2} E \rightarrow \xi_{*} \mathcal{O}_{B}\left(2 C_{1}\right) \rightarrow S^{2} E \rightarrow 0
$$

In addition, from (2), $\xi$ must be factored into

$$
\xi: B \stackrel{\sigma}{\longrightarrow} C^{\prime} \stackrel{\eta}{\longrightarrow} C
$$

for some unramified cyclic triple covering $\eta$ and unramified double covering $\sigma$ [3, Proposition 11.4.3].

First, notice that $\eta^{*} E$ is stable. For, otherwise, its destabilizing line bundle $\eta^{*} E \rightarrow S$ of degree 0 gives a morphism $\phi: C^{\prime} \rightarrow X$ over $C$ and an $m$-section $\phi\left(C^{\prime}\right) \equiv m C_{1}+\operatorname{deg} \phi^{*} \mathcal{O}_{X}\left(C_{1}\right) \cdot f=m C_{1}+\operatorname{deg} S \cdot f=m C_{1}$ on $X$ for some $m \mid 3$. It contradicts the assumption that $S^{3} E$ is not destabilized by a line subbundle.

Next, note that $\eta^{*} E \cong \sigma_{*} \mathcal{O}_{B}\left(C_{1}\right)$. Because $B \sim 6 C_{1}$ is effective, by pushing forward the exact sequence

$$
0 \rightarrow \mathcal{O}_{X}\left(-5 C_{1}\right) \rightarrow \mathcal{O}_{X}\left(C_{1}\right) \rightarrow \mathcal{O}_{B}\left(C_{1}\right) \rightarrow 0
$$

on $X$ to $C$, we obtain an injection $E \rightarrow \xi_{*} \mathcal{O}_{B}\left(C_{1}\right)=\eta_{*}\left(\sigma_{*} \mathcal{O}_{B}\left(C_{1}\right)\right)$. Then it gives a nonzero morphism $\eta^{*} E \rightarrow \sigma_{*} \mathcal{O}_{B}\left(C_{1}\right)$ on $C^{\prime}$, which is an isomorphism as $\eta^{*} E$ is stable and $\operatorname{deg} \eta^{*} E=\operatorname{deg} \mathcal{O}_{B}\left(C_{1}\right)=0$.

Then we check that $\mathcal{O}_{B}\left(2 C_{1}\right) \in \operatorname{Pr}\left(B / C^{\prime}\right)$. From $\operatorname{det} \sigma_{*} \mathcal{O}_{B}\left(C_{1}\right) \cong \operatorname{det} \sigma_{*} \mathcal{O}_{B} \otimes \operatorname{Nm}_{B / C^{\prime}}\left(\mathcal{O}_{B}\left(C_{1}\right)\right)$ and $\left(\operatorname{det} \sigma_{*} \mathcal{O}_{B}\right)^{2}=\mathcal{O}_{C^{\prime}}$ as $\sigma$ is unramified, we can observe that

$$
\mathrm{Nm}_{B / C^{\prime}}\left(\mathcal{O}_{B}\left(2 C_{1}\right)\right)=\left\{\mathrm{Nm}_{B / C^{\prime}}\left(\mathcal{O}_{B}\left(C_{1}\right)\right)\right\}^{2} \cong\left(\operatorname{det} \sigma_{*} \mathcal{O}_{B}\left(C_{1}\right)\right)^{2}
$$

Since $\sigma_{*} \mathcal{O}_{B}\left(C_{1}\right) \cong \eta^{*} E$ and $\operatorname{det} \eta^{*} E=\eta^{*} \operatorname{det} E=\mathcal{O}_{C^{\prime}}$, we get $\operatorname{Nm}_{B / C^{\prime}}\left(\mathcal{O}_{B}\left(2 C_{1}\right)\right)=\mathcal{O}_{C^{\prime}}$.

Also, we deduce that $\mathcal{O}_{B}\left(4 C_{1}\right)=\mathcal{O}_{B}$ from (3). Indeed, if $\mathcal{O}_{B}\left(C_{1}\right)=\mathcal{O}_{B}\left(3 C_{1}\right)$, then $\mathcal{O}_{B}\left(2 C_{1}\right)=\mathcal{O}_{B}$. Otherwise, if $\mathcal{O}_{B}\left(C_{1}\right) \neq \mathcal{O}_{B}\left(3 C_{1}\right)$, then $\eta^{*} E=\mathcal{O}_{B}\left(C_{1}\right) \oplus \mathcal{O}_{B}\left(3 C_{1}\right)$ and $\mathcal{O}_{B}\left(4 C_{1}\right)=\operatorname{det} \eta^{*} E=\mathcal{O}_{B}$.

Now we have $\mathcal{O}_{B}\left(2 C_{1}\right) \in \operatorname{Pr}\left(B / C^{\prime}\right)$ and $\mathcal{O}_{B}\left(2 C_{1}\right) \in J_{2}(B)$. Thus $\sigma_{*} \mathcal{O}_{B}\left(2 C_{1}\right)$ is orthogonal with values in $\mathcal{O}_{C^{\prime}}$, and $\mathcal{O}_{B}\left(2 C_{1}\right) \in \sigma^{*} J^{0}\left(C^{\prime}\right)$ by Proposition 2.2. Due to Proposition 2.1, we can see that $\sigma_{*} \mathcal{O}_{B}\left(2 C_{1}\right)$ is strictly semi-stable, and by Remark 2.4, $\sigma_{*} \mathcal{O}_{B}\left(2 C_{1}\right)=M \oplus N$ for some $M, N \in J_{2}\left(C^{\prime}\right)$. Therefore, $S^{2} E \cong \eta_{*} M$ is obtained from the stability of $S^{2} E$ and the following exact sequence from (4).

$$
0 \rightarrow S^{2} E \rightarrow \eta_{*}\left(\sigma_{*} \mathcal{O}_{B}\left(2 C_{1}\right)\right) \cong\left(\eta_{*} M\right) \oplus\left(\eta_{*} N\right) \rightarrow S^{2} E \rightarrow 0
$$

Finally, $M \in \eta^{*} J^{0}(C)$ if and only if $S^{2} E=\eta_{*} M$ is strictly semi-stable as in Example 3.5.

This is the end of the classification of $E \in \mathcal{S U}_{C}\left(2, \mathcal{O}_{C}\right)$ with stable $S^{2} E$ and strictly semi-stable $S^{3} E$; by Theorem 3.11, they are only given as in Example 3.5, and the number of such $E$ is finite according to Proposition 3.6 and the argument preceding the proposition. Recall that we observed in Theorem 3.3 that if $S^{3} E$ is destabilized by a line subbundle, then $S^{2} E$ is necessarily not stable. 
If $S^{2} E$ is stable, then $S^{3} E$ is not stable if and only if $S^{3} E$ is destabilized by subbundles $E \otimes L^{\mp 1} \rightarrow S^{3} E$ for some line bundle $L$ due to Proposition 3.9. Moreover, from an exact sequence

$$
0 \rightarrow \operatorname{Hom}\left(L^{\mp 1}, S^{4} E\right) \rightarrow \operatorname{Hom}\left(E \otimes L^{\mp 1}, S^{3} E\right) \rightarrow \operatorname{Hom}\left(L^{\mp 1}, S^{2} E\right)
$$

of Proposition 3.1 with $k=3$, we can see that $S^{3} E$ is destabilized by subbundles $E \otimes L^{\mp 1}$ if and only if $S^{4} E$ is destabilized by line subbundles $L^{\mp 1} \rightarrow S^{4} E$ when $S^{2} E$ is stable so that $\operatorname{Hom}\left(L^{\mp 1}, S^{2} E\right)=0$. Therefore, we have the following corollary under the assumption on the stability of $S^{2} E$.

Corollary 3.12. If $S^{2} E$ is stable, then $S^{3} E$ is strictly semi-stable if and only if $S^{4} E$ is destabilized by a line subbundle, and the number of such $E \in \mathcal{S U}_{C}\left(2, \mathcal{O}_{C}\right)$ is finite. In particular, there exist only a finite number of ruled surfaces $X=\mathbb{P}_{C}(E)$ for stable $E$ with even degree where $X$ contains a 4 -section of zero self-intersection whereas it has no such $m$-section for any $m<4$.

\section{Stability of Higher Symmetric Powers}

4.1. Finiteness Result. In the previous sections, we prove that the family of $E \in \mathcal{S U}_{C}\left(2, \mathcal{O}_{C}\right)$ with strictly semi-stable $S^{2} E$ has positive dimension, but there are only finitely many $E \in \mathcal{S U}_{C}\left(2, \mathcal{O}_{C}\right)$ with strictly semi-stable $S^{3} E$ outside this family. We will strengthen the result as showing that there are only a finite number of $E \in \mathcal{S U}_{C}\left(2, \mathcal{O}_{C}\right)$ where $S^{2} E$ is stable but $S^{k} E$ is not stable for some $k \geq 3$.

Throughout this and the next subsection, we assume that

- $E$ is a stable vector bundle on $C$ of rank 2 with trivial determinant,

- $X=\mathbb{P}_{C}(E)$ is the ruled surface $\Pi: \mathbb{P}_{C}(E) \rightarrow C$,

- $C_{1}$ is a unisecant divisor on $X$ satisfying $\Pi_{*} \mathcal{O}_{X}\left(C_{1}\right)=E$, and

- $\pi=\left.\Pi\right|_{D}: D \rightarrow C$ is the $k$-covering induced from a $k$-section $D$ on $X$.

Then, by Remark 2.6, $\pi$ is unramified if and only if $D^{2}=0$. Also, from the exact sequence (3.1) in the proof of Proposition 3.1 and its dual sequence with the self-duality $E^{\vee} \cong E$, we obtain two injections

$$
S^{k} E \rightarrow S^{k+1} E \otimes E \quad \text { and } \quad S^{k} E \rightarrow S^{k-1} E \otimes E .
$$

Thus if there is a subbundle $V \rightarrow S^{k} E$, then it induces injections $V \rightarrow S^{k+1} E \otimes E$ and $V \rightarrow S^{k-1} E \otimes E$, which respectively induce nonzero morphisms

$$
V \otimes E \rightarrow S^{k-1} E \quad \text { and } \quad V \otimes E \rightarrow S^{k+1} E
$$

due to $\operatorname{Hom}\left(V \otimes E, S^{k \pm 1} E\right)=H^{0}\left((V \otimes E)^{\vee} \otimes S^{k \pm 1} E\right)=H^{0}\left(V^{\vee} \otimes S^{k \pm 1} E \otimes E\right)=\operatorname{Hom}\left(V, S^{k \pm 1} E \otimes E\right)$.

Proposition 4.1. Let $k \geq 2$. If $S^{k-1} E$ is stable, then $S^{k} E$ is not destabilized by a subbundle $V \rightarrow S^{k} E$ of $\operatorname{rk} V<\frac{k}{2}$. Moreover, when $S^{k-1} E$ is stable, $S^{k} E$ is not stable if and only if it is destabilized by a stable subbundle $V \rightarrow S^{k} E$ of $\operatorname{rk} V=\frac{k+1}{2}$ if $k$ is odd or $\operatorname{rk} V=\frac{k}{2}$ if $k$ is even.

Proof. Assume that $S^{k} E$ is destabilized by a subbundle $V \rightarrow S^{k} E$ of $\operatorname{rk} V=r$ and $\operatorname{deg} V=0$. As (4.1), the injection $V \rightarrow S^{k} E$ gives a nonzero morphism $V \otimes E \rightarrow S^{k-1} E$. Since $\operatorname{deg}(V \otimes E)=0$ and $S^{k-1} E$ is assumed to be stable, the morphism must be surjective, so $k=\operatorname{rk} S^{k-1} E \leq \operatorname{rk}(V \otimes E)=2 r$. That is, $\frac{k+1}{2} \leq r$ if $k$ is odd or $\frac{k}{2} \leq r$ otherwise.

Due to Remark 1.1, when $S^{k} E$ is not stable, we can find a destabilizing subbundle $V \rightarrow S^{k} E$ of rank $r \leq \frac{k+1}{2}$ if $k$ is odd or $r \leq \frac{k}{2}$ otherwise, and each equality holds if $S^{k-1} E$ is stable as shown above. Because a destabilizing subbundle of $V$ destabilizes $S^{k} E$ in a smaller rank if it exists, $V$ must be stable. 
Theorem 4.2. Let $k \geq 2$. If $S^{k-1} E$ is stable but $S^{k} E$ is not stable, then $E$ corresponds to one of the following cases.

(1) $S^{2} E$ is destabilized by a subbundle of rank 1

(2) $S^{3} E$ is destabilized by a subbundle of rank 2

(3) $S^{4} E$ is destabilized by a subbundle of rank 2

(4) $S^{6} E$ is destabilized by a subbundle of rank 3

In particular, $S^{k} E$ is stable for every $k \geq 2$ if $S^{m} E$ is stable for all $m \leq 6$.

Proof. From the proof of Proposition 4.1, we observe that if $S^{k-1} E$ is stable but $S^{k} E$ is not stable, then there exist an injection $V \rightarrow S^{k} E$ and a surjection $V \otimes E \rightarrow S^{k-1} E$ for some stable vector bundle $V$ of degree 0 and rank $r=\frac{k+1}{2}$ if $k$ is odd or $r=\frac{k}{2}$ otherwise.

If $k$ is odd, then $2 r=k+1$, and the surjection $V \otimes E \rightarrow S^{k-1} E$ gives the exact sequence

$$
0 \rightarrow L \rightarrow V \otimes E \rightarrow S^{k-1} E \rightarrow 0
$$

for some line bundle $L$ of degree 0 , and the injection $L \rightarrow V \otimes E$ induces a nonzero morphism $L \otimes E \cong$ $L \otimes E^{\vee} \rightarrow V$. This is possible only if $r=\operatorname{rk} V=2$ because $L \otimes E$ and $V$ are stable of the same degree. Thus it implies that $k=3$.

Otherwise, if $k$ is even, then $2 r=k$, so the surjection $V \otimes E \rightarrow S^{k-1} E$ becomes an isomorphism $V \otimes E \cong S^{k-1} E$. On the other hand, the injection $V \rightarrow S^{k} E$ gives a nonzero morphism $V \otimes E \rightarrow S^{k+1} E$ as (4.1). By taking the dual, a nonzero morphism $S^{k+1} E \cong\left(S^{k+1} E\right)^{\vee} \rightarrow(V \otimes E)^{\vee} \cong\left(S^{k-1} E\right)^{\vee} \cong S^{k-1} E$ is obtained, which must be surjective due to the stability of $S^{k-1} E$. Then, from the exact sequence

$$
0 \rightarrow Q \rightarrow S^{k+1} E \rightarrow S^{k-1} E \rightarrow 0
$$

for some vector bundle $Q$ of rank 2 and degree 0 , we can see that $S^{k+1} E$ is destabilized by a subbundle $Q \rightarrow S^{k+1} E$ of rank 2. Using the injections $S^{k+1} E \rightarrow S^{k} E \otimes E$ and $S^{k} E \rightarrow S^{k-1} E \otimes E$ twisted by $E$, the injection $Q \rightarrow S^{k+1} E$ induces an injection $Q \rightarrow S^{k-1} E \otimes E \otimes E$, and it yields a nonzero morphism $Q \otimes E \otimes E \rightarrow S^{k-1} E$ due to $\operatorname{Hom}\left(Q \otimes E \otimes E, S^{k-1} E\right)=\operatorname{Hom}\left(Q, S^{k-1} E \otimes E \otimes E\right)$ similar to (4.1). Since $S^{k-1} E$ is assumed to be stable, the morphism

$$
Q \oplus\left(Q \otimes S^{2} E\right) \cong Q \otimes\left(\mathcal{O}_{C} \oplus S^{2} E\right) \cong Q \otimes E \otimes E \rightarrow S^{k-1} E
$$

is necessarily surjective, and again from the stability of $S^{k-1} E$, one of the morphisms $Q \rightarrow S^{k-1} E$ or $Q \otimes S^{2} E \rightarrow S^{k-1} E$ must be surjective. Therefore, $k=\operatorname{rk} S^{k-1} E \leq \max \left\{\operatorname{rk} Q, \operatorname{rk}\left(Q \otimes S^{2} E\right)\right\}=6$.

Notice that cases (1) and (2) in the theorem are already studied in Section 2 and 3 respectively. We will show that there are only a finite number of $E$ in cases (3) and (4) as in case (2).

Proposition 4.3. Let $k \geq 3$. Assume that there is a $k$-section $\pi: D \rightarrow C$ of zero self-intersection on $X$ and no such $m$-section for any $m<k$. Then there is a surjection $\pi^{*} E \rightarrow R$ for some $R \in J_{2(k-1)(k-2)}(D)$.

Proof. Let $D \sim k C_{1}+\mathfrak{b} f$ for some $\mathfrak{b} \in \operatorname{Pic}(C)$, necessarily satisfying $\operatorname{deg} \mathfrak{b}=0$. Due to Lemma 3.7 and Remark 2.6. $D$ is smooth and $\pi: D \rightarrow C$ is unramified. Also, note that the morphism $\phi: D \rightarrow X$ over $C$ gives a surjection $\pi^{*} E \rightarrow R$ on $D$ for $R=\phi^{*} \mathcal{O}_{X}\left(C_{1}\right)=\mathcal{O}_{D}\left(C_{1}\right)$ with $\operatorname{deg} R=D \cdot C_{1}=\left(k C_{1}+\mathfrak{b} f\right) \cdot C_{1}=0$.

By applying [14, I: p. 248] and comparing the determinants after pushing forward the exact sequence

$$
0 \rightarrow \mathcal{O}_{X}\left(-k C_{1}-\mathfrak{b} f\right) \rightarrow \mathcal{O}_{X} \rightarrow \mathcal{O}_{D} \rightarrow 0
$$


on $X$ to $C$, we obtain that $L^{-2(k-1)}=\left(\operatorname{det}\left(\mathcal{O}_{C} \oplus\left(S^{k-2} E \otimes L^{-1}\right)\right)\right)^{2}=\left(\operatorname{det} \pi_{*} \mathcal{O}_{D}\right)^{2}=\mathcal{O}_{C}$ for $L=\mathcal{O}_{C}(\mathfrak{b})$ because $\pi$ is unramified. Then, by Proposition 2.7, we have

$$
R^{k-2}=\mathcal{O}_{D}\left((k-2) C_{1}\right)=\mathcal{O}_{D}(-\mathfrak{b} f)=\pi^{*} L^{-1},
$$

and hence $R^{2(k-1)(k-2)}=\left(\pi^{*} L^{-1}\right)^{2(k-1)}=\pi^{*} L^{-2(k-1)}=\pi^{*} \mathcal{O}_{C}=\mathcal{O}_{D}$.

Remark 4.4. In the proof of Proposition 4.3 , when $E$ has trivial determinant and $S^{k} E$ is destabilized by a quotient line bundle $S^{k} E \rightarrow L$, it is shown that $L^{2(k-1)}=\mathcal{O}_{C}$. This is a generalization of Remark 2.3 , It is also compatible with $L^{4}=\mathcal{O}_{C}$ for $k=3$ (Theorem 3.3) and $L^{3}=\mathcal{O}_{C}$ for $k=4$ (Proposition 3.9).

Corollary 4.5. Let $k \geq 3$. Then there exist at most finitely many $E \in \mathcal{S U}_{C}\left(2, \mathcal{O}_{C}\right)$ such that $S^{k} E$ is destabilized by a line subbundle but $S^{m} E$ is not destabilized by a line subbundle for any $m<k$.

Proof. The assertion follows from Proposition 4.3 and the finiteness of the following data.

- the unramified $k$-coverings $\pi: D \rightarrow C$

- the torsion line bundles on $D$ of order $2(k-1)(k-2)$

- the direct summands of graded bundle of a Jordan-Hölder filtration associated to $\pi_{*} R$

By the adjoint property, a surjection $\pi^{*} E \rightarrow R$ gives a nonzero morphism $E \rightarrow \pi_{*} R$, so we can deduce that $E$ is a subbundle of $\pi_{*} R$ as $E$ is stable and $\operatorname{deg} E=\operatorname{deg} \pi_{*} R=0$.

Lemma 4.6. There exists the following exact sequence on $C$.

$$
0 \rightarrow S^{n-1} E \otimes S^{m-1} E \rightarrow S^{n} E \otimes S^{m} E \rightarrow S^{n+m} E \rightarrow 0
$$

Proof. By restricting the natural morphism $\Pi^{*} \Pi_{*} \mathcal{O}_{X}\left(m C_{1}\right) \rightarrow \mathcal{O}_{X}\left(m C_{1}\right)$ to the fiber $P f=\Pi^{-1}(P)$ of $X$, we have the morphism $\left.\left.\Pi^{*} \Pi_{*} \mathcal{O}_{X}\left(m C_{1}\right)\right|_{P f} \rightarrow \mathcal{O}_{X}\left(m C_{1}\right)\right|_{P f}$ over each $P \in C$, which is identified with the evaluation morphism $H^{0}\left(\mathbb{P}^{1}, \mathcal{O}_{\mathbb{P}^{1}}\left(m C_{1}\right)\right) \otimes \mathcal{O}_{\mathbb{P}^{1}} \rightarrow \mathcal{O}_{\mathbb{P}^{1}}(m)$ from Grauert's theorem,

$$
\left.\Pi^{*} \Pi_{*} \mathcal{O}_{X}\left(m C_{1}\right)\right|_{P f} \cong\left(\Pi_{*} \mathcal{O}_{X}\left(m C_{1}\right) \otimes \mathbb{C}(P)\right) \otimes \mathcal{O}_{P f} \cong H^{0}\left(P f,\left.\mathcal{O}_{X}\left(m C_{1}\right)\right|_{P f}\right) \otimes \mathcal{O}_{P f}
$$

As $\left.\mathcal{O}_{X}\left(m C_{1}\right)\right|_{P f} \cong \mathcal{O}_{\mathbb{P}^{1}}(m)$ is generated by its global sections, the evaluation morphism is surjective over each $P \in C$, so the morphism $\Pi^{*} \Pi_{*} \mathcal{O}_{X}\left(m C_{1}\right) \rightarrow \mathcal{O}_{X}\left(m C_{1}\right)$ is surjective on $X$ by Nakayama's lemma. Thus we have the exact sequence

$$
0 \rightarrow K \otimes \mathcal{O}_{X}\left(-C_{1}\right) \rightarrow \Pi^{*} \Pi_{*} \mathcal{O}_{X}\left(m C_{1}\right) \rightarrow \mathcal{O}_{X}\left(m C_{1}\right) \rightarrow 0
$$

for some vector bundle $K$ on $X$ of rank $m$ and $c_{1}(K)=\mathcal{O}_{X}$ which satisfies $\left.K\right|_{P f} \cong \mathcal{O}_{P f}{ }^{\oplus m}$ for each $P \in C$. Indeed, note that $\left.K\right|_{P f} \otimes \mathcal{O}_{\mathbb{P}^{1}}(-1) \cong \mathcal{O}_{\mathbb{P}^{1}}\left(a_{1}\right) \oplus \cdots \oplus \mathcal{O}_{\mathbb{P}^{1}}\left(a_{m}\right)$ for some $a_{i} \leq 0$ with $a_{1}+\cdots+a_{m}=-m$, and from the associated long exact sequence of cohomology groups,

$$
0 \rightarrow H^{0}\left(\mathbb{P}^{1},\left.K\right|_{P f} \otimes \mathcal{O}_{\mathbb{P}^{1}}(-1)\right) \rightarrow H^{0}\left(\mathbb{P}^{1}, H^{0}\left(\mathbb{P}^{1}, \mathcal{O}_{\mathbb{P}^{1}}\left(m C_{1}\right)\right) \otimes \mathcal{O}_{\mathbb{P}^{1}}\right) \rightarrow H^{0}\left(\mathbb{P}^{1}, \mathcal{O}_{\mathbb{P}^{1}}(m)\right),
$$

we can observe that $H^{0}\left(\left.K\right|_{P f} \otimes \mathcal{O}_{\mathbb{P}^{1}}(-1)\right)=H^{0}\left(\mathcal{O}_{\mathbb{P}^{1}}\left(a_{1}\right)\right) \oplus \cdots \oplus H^{0}\left(\mathcal{O}_{\mathbb{P}^{1}}\left(a_{m}\right)\right)=0$ because the last morphism is bijective. Thus $a_{i} \leq-1$, and we have $a_{i}=-1$ for all $i=1, \ldots, m$.

By pushing forward exact sequence (4.3) on $X$ to $C$ after twisting by $\mathcal{O}_{X}\left(-C_{1}\right)$, we have

$$
S^{m-1} E \cong R^{1} \Pi_{*}\left(K \otimes \mathcal{O}_{X}\left(-2 C_{1}\right)\right) \cong\left(\Pi_{*} K\right)^{\vee}
$$

from $\omega_{X / C} \cong \mathcal{O}_{X}\left(-2 C_{1}\right)$ and the relative Serre duality. Since $\Pi^{*} S^{m-1} E \cong \Pi^{*}\left(S^{m-1} E\right)^{\vee} \cong \Pi^{*} \Pi_{*} K$, we get a morphism $\Pi^{*} S^{m-1} E \cong \Pi^{*} \Pi_{*} K \rightarrow K$ on $X$ where the latter morphism $\Pi^{*} \Pi_{*} K \rightarrow K$ is surjective 
as $\left.K\right|_{P f} \cong \mathcal{O}_{P f}^{\oplus m}$ is generated by its global sections for all $P \in C$. Because $S^{m-1} E$ is of rank $m$, we have $K \cong \Pi^{*} S^{m-1} E$, and there exists the following exact sequence on $X$.

$$
0 \rightarrow \Pi^{*} S^{m-1} E \otimes \mathcal{O}_{X}\left(-C_{1}\right) \rightarrow \Pi^{*} S^{m} E \rightarrow \mathcal{O}_{X}\left(m C_{1}\right) \rightarrow 0
$$

Therefore, we obtain the desired exact sequence by pushing forward the above sequence to $C$ after twisting by $\mathcal{O}_{X}\left(n C_{1}\right)$.

Remark 4.7. The reviewer points out that as $E$ is the associated vector bundle of some principal $\mathrm{SL}_{2} \mathbb{C}$-bundle $P \rightarrow C, S^{k} E$ is the associated vector bundle of $P\left(S^{k} V\right)$ where $V=\mathbb{C}^{2}$ is the standard representation of $\mathrm{SL}_{2} \mathbb{C}$, hence Lemma 4.6 is a consequence of [10, Exercise 11.11].

Theorem 4.8. If $S^{2} E$ is stable, then every $S^{k} E$ is stable except for finitely many $E \in \mathcal{S U}_{C}\left(2, \mathcal{O}_{C}\right)$.

Proof. Assume that $S^{k} E$ is not stable and $S^{m} E$ is stable for all $m<k$. By Theorem 4.2, it remains to treat $k=3,4$, and 6 . For $k=3$, we know from Corollary 3.12 that $S^{4} E$ is destabilized by a line subbundle. In the cases of even $k=4,6$, we can observe from exact sequence (4.2) in the proof of Theorem 4.2 that there exists a surjection $S^{k+1} E \rightarrow S^{k-1} E$ so that $H^{0}\left(S^{k-1} E \otimes S^{k+1} E\right)=H^{0}\left(\left(S^{k+1} E\right)^{\vee} \otimes S^{k-1} E\right) \neq 0$. By taking global sections of the exact sequence of Lemma 4.6 with $n=k-1$ and $m=k+1$,

$$
0 \rightarrow S^{k-2} E \otimes S^{k} E \rightarrow S^{k-1} E \otimes S^{k+1} E \rightarrow S^{2 k} E \rightarrow 0,
$$

we have either $H^{0}\left(S^{k-2} E \otimes S^{k} E\right) \neq 0$ or $H^{0}\left(S^{2 k} E\right) \neq 0$ from $H^{0}\left(S^{k-1} E \otimes S^{k+1} E\right) \neq 0$. If $H^{0}\left(S^{2 k} E\right)=0$, then $H^{0}\left(S^{k-2} E \otimes S^{k} E\right) \neq 0$, and in this case, by taking global sections of the exact sequence of the same Lemma with $n=k-2$ and $m=k$,

$$
0 \rightarrow S^{k-3} E \otimes S^{k-1} E \rightarrow S^{k-2} E \otimes S^{k} E \rightarrow S^{2 k-2} E \rightarrow 0
$$

we have either $H^{0}\left(S^{k-3} E \otimes S^{k-1} E\right) \neq 0$ or $H^{0}\left(S^{2 k-2} E\right) \neq 0$, where the former is impossible because, if then, it gives a nonzero morphism $S^{k-3} E \cong\left(S^{k-3} E\right)^{\vee} \rightarrow S^{k-1} E$ which destabilizes $S^{k-1} E$. Therefore, from the assumption that $H^{0}\left(S^{k-1} E \otimes S^{k+1} E\right) \neq 0$, we obtain either $H^{0}\left(S^{2 k} E\right) \neq 0$ or $H^{0}\left(S^{2 k-2} E\right) \neq 0$. In other words, either $S^{2 k} E$ or $S^{2 k-2} E$ is destabilized by a line subbundle.

Therefore, if $S^{2} E$ is stable but $S^{k} E$ is not stable for some $k \geq 3$, then $S^{l} E$ is destabilized by a line subbundle for some $4 \leq l \leq 12$. However, since $S^{2} E$ is stable, $S^{3} E$ cannot be destabilized by a line subbundle due to Proposition 3.1 Thus $E$ is contained in the collection

$$
\bigcup_{l=4}^{12}\left\{E \in \mathcal{S U}_{C}\left(2, \mathcal{O}_{C}\right) \mid S^{l} E \text { is destabilized by a line subbundle but } S^{m} E \text { is not for any } m<l\right\}
$$

which must be finite thanks to Corollary 4.5 .

As the locus of strictly semi-stable $E \in \mathcal{S U}_{C}\left(2, \mathcal{O}_{C}\right)$ is given by the image of a map from the Jacobian variety, the locus is closed. Also, the locus of $E \in \mathcal{S U}_{C}\left(2, \mathcal{O}_{C}\right)$ with strictly semi-stable $S^{2} E$ is closed in $\mathcal{S U}_{C}\left(2, \mathcal{O}_{C}\right)$ because it is the union of finitely many images of maps from the Prym varieties. Since a finite union of proper closed subsets is still a proper closed subset, the theorem gives the following fact.

Corollary 4.9. If $E$ is stable, then every $S^{k} E$ is stable for general $E \in \mathcal{S U}_{C}\left(2, \mathcal{O}_{C}\right)$.

4.2. Relation with Étale Triviality. $E$ is said to be étale-trivial if there exists an unramified finite covering $\eta: C^{\prime} \rightarrow C$ with $\eta^{*} E=\mathcal{O}_{C^{\prime}} \oplus \mathcal{O}_{C^{\prime}}$. Thus if $E$ is étale-trivial, then the trivialization induces a morphism $\phi: C^{\prime} \rightarrow X$ over $C$ with $\operatorname{deg} \phi^{*} \mathcal{O}_{X}\left(C_{1}\right)=0$ and $\phi\left(C^{\prime}\right) \equiv k C_{1}+\operatorname{deg} \phi^{*} \mathcal{O}_{X}\left(C_{1}\right) f=k C_{1}$ for some $k>0$. Therefore, the image $\phi\left(C^{\prime}\right)$ becomes a $k$-section of zero self-intersection on $X$ so that $S^{k} E$ is destabilized by a line subbundle. We will see when the converse holds. 
Lemma 4.10. Assume that $E$ splits over an unramified finite covering $\pi: D \rightarrow C$ as $\pi^{*} E=R^{-1} \oplus R$ for some line bundle $R$ on $D$. Then $E$ is étale-trivial if and only if $R \in J_{m}(D)$ for some $m>0$.

Proof. Recall that we assumed $\operatorname{det} E=\mathcal{O}_{C}$. So if $\pi^{*} E$ splits, then it must be of the form $\pi^{*} E=R^{-1} \oplus R$. If $E$ is étale-trivial, then there exists an unramified finite covering $\eta: C^{\prime} \rightarrow C$ over which $E$ is trivialized. Let $D^{\prime}=D \times{ }_{C} C^{\prime}$ be the covering of $C$ which gives the following commutative diagram.

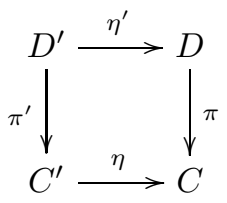

From

$$
\eta^{\prime *}\left(R^{-1} \oplus R\right)=\left(\pi \circ \eta^{\prime}\right)^{*} E=\left(\eta \circ \pi^{\prime}\right)^{*} E=\pi^{\prime *}\left(\mathcal{O}_{C^{\prime}} \oplus \mathcal{O}_{C^{\prime}}\right)=\mathcal{O}_{D^{\prime}} \oplus \mathcal{O}_{D^{\prime}}
$$

we can observe that $\eta^{\prime *} R=\mathcal{O}_{D^{\prime}}$. It is possible only if $R$ is a torsion line bundle [3, Proposition 11.4.3].

The converse holds by composing $\pi$ with a cyclic covering over which $R$ is trivialized.

If $E$ is stable but $S^{2} E$ is not stable, then, by Proposition 2.5, there exists a nontrivial unramified double covering $\pi: B \rightarrow C$ and $E=\pi_{*} R$ for some $R \in J^{0}(B)$. If $R \neq \iota^{*} R$, then $\pi^{*} E$ splits as $\pi^{*} E=\iota^{*} R \oplus R$ because $\pi^{*} E$ is invariant under the involution $\iota: B \rightarrow B$ induced by $\pi$. Otherwise, if $R=\iota^{*} R$, then $R \in \pi^{*} J^{0}(C)$, so $E$ already splits on $C$ by Proposition 2.1. Hence $\pi^{*} E$ splits over an unramified double covering $\pi: B \rightarrow C$ whenever $S^{2} E$ is not stable. Since $\operatorname{det} E=\mathcal{O}_{C}$, it must be of the form $\pi^{*} E=R^{-1} \oplus R$. Therefore, we have the following observation applying the previous lemma.

Remark 4.11. If $E$ is stable but $S^{2} E$ is not stable, then $E$ is étale-trivial if and only if $E=\pi_{*} R$ for some nontrivial unramified double covering $\pi: B \rightarrow C$ and $R \in J_{m}(B)$ for some $m>0$.

Lemma 4.12. Let $k \geq 3$. Assume that $S^{k} E$ is destabilized by a line subbundle $L^{-1} \rightarrow S^{k} E$ but $S^{m} E$ is not destabilized by a line subbundle for any $m<k$. Then $S^{k-2} E \otimes L^{-1} \cong S^{k-2} E \otimes L$.

Proof. Let $D \sim k C_{1}+\mathfrak{b} f$ be the $k$-section on $X$ corresponding to the line subbundle $L^{-1} \rightarrow S^{k} E$. Then $D$ is irreducible and reduced by Lemma 3.7. By pushing forward the exact sequences

$$
\begin{aligned}
0 & \rightarrow \mathcal{O}_{X}\left(-k C_{1}-\mathfrak{b} f\right) \rightarrow \mathcal{O}_{X} \rightarrow \mathcal{O}_{D} \rightarrow 0, \\
0 \rightarrow \mathcal{O}_{X}\left(-2 C_{1}\right) & \rightarrow \mathcal{O}_{X}\left((k-2) C_{1}+\mathfrak{b} f\right) \rightarrow \mathcal{O}_{D}\left((k-2) C_{1}+\mathfrak{b} f\right) \rightarrow 0
\end{aligned}
$$

on $X$ to $C$ and using $\mathcal{O}_{D}\left((k-2) C_{1}+\mathfrak{b} f\right)=\mathcal{O}_{D}$ from Proposition 2.7, we get the following exact sequences.

$$
\begin{gathered}
0 \rightarrow \mathcal{O}_{C} \rightarrow \pi_{*} \mathcal{O}_{D} \rightarrow S^{k-2} E \otimes L^{-1} \rightarrow 0 \\
0 \rightarrow S^{k-2} E \otimes L
\end{gathered}
$$

Since $D$ is smooth by Remark 2.6 $\pi_{*} \mathcal{O}_{D}$ has a splitting of the injection $\mathcal{O}_{C} \rightarrow \pi_{*} \mathcal{O}_{D}$ [14, I: p. 248], which indeed follows from the hypothesis on $S^{m} E$ for $m<k$. Therefore, $\mathcal{O}_{C} \oplus\left(S^{k-2} E \otimes L^{-1}\right) \cong \pi_{*} \mathcal{O}_{D} \cong$ $\mathcal{O}_{C} \oplus\left(S^{k-2} E \otimes L\right)$, and hence $S^{k-2} E \otimes L^{-1} \cong S^{k-2} E \otimes L$.

Theorem 4.13. Let $k \geq 3$. If there is a $k$-section $\pi: D \rightarrow C$ of zero self-intersection on $X=\mathbb{P}_{C}(E)$ and no such $m$-section for any $m<k$, then $\pi^{*} E=R^{-1} \oplus R$ for some $R \in J_{2(k-1)(k-2)}(D)$. Moreover, if $S^{2} E$ is stable, then $E$ is étale-trivial if and only if $S^{k} E$ is destabilized by a line subbundle for some $k \geq 3$.

Proof. Let $L^{-1} \rightarrow S^{k} E$ be the destabilizing line subbundle corresponding to the $k$-section $D \sim k C_{1}+\mathfrak{b} f$ on $X$ for $L=\mathcal{O}_{C}(\mathfrak{b})$. Then $D$ is irreducible and reduced by Lemma 3.7 and so $\pi: D \rightarrow C$ is unramified 
by Remark 2.6. Let $R=\mathcal{O}_{D}\left(C_{1}\right)$. We observe in Proposition 4.3 that $R$ is a torsion line bundle and there exists a surjection $\pi^{*} E \rightarrow R$. In order to conclude that $\pi^{*} E=R^{-1} \oplus R$, it remains to show that there exists another surjection $\pi^{*} E \rightarrow R^{-1}$ and $R^{-1} \neq R$.

We first claim that $R^{-1} \neq R$. Suppose $\mathcal{O}_{D}\left(2 C_{1}\right)=R^{2}=\mathcal{O}_{D}$. By pushing forward the exact sequence

$$
0 \rightarrow \mathcal{O}_{X}\left(-(k+4) C_{1}-\mathfrak{b} f\right) \rightarrow \mathcal{O}_{X}\left(-4 C_{1}\right) \rightarrow \mathcal{O}_{D}\left(-4 C_{1}\right) \rightarrow 0
$$

on $X$ to $C$, we have an injection $\pi_{*} \mathcal{O}_{D}=\pi_{*} \mathcal{O}_{D}\left(-4 C_{1}\right) \rightarrow R^{1} \pi_{*} \mathcal{O}_{X}\left(-(k+4) C_{1}-\mathfrak{b} f\right)=S^{k+2} E \otimes L^{-1}$. Since $H^{0}\left(\pi_{*} \mathcal{O}_{D}\right) \neq 0$ and $H^{0}\left(\pi_{*} \mathcal{O}_{D}\right) \subseteq H^{0}\left(S^{k+2} E \otimes L^{-1}\right)$, we get $H^{0}\left(S^{k+2} E \otimes L^{-1}\right) \neq 0$. So there exists a $(k+2)$-section $B \sim(k+2) C_{1}-\mathfrak{b} f$, and we have the following exact sequence on $X$.

$$
0 \rightarrow \mathcal{O}_{X}\left(-(k+2) C_{1}+\mathfrak{b} f\right) \rightarrow \mathcal{O}_{X} \rightarrow \mathcal{O}_{B} \rightarrow 0
$$

By pushing forward the sequence to $C$, we obtain the isomorphism $\pi_{*} \mathcal{O}_{B} \cong \mathcal{O}_{C} \oplus\left(S^{k} E \otimes L\right)$ thanks to [14, I: p. 248] if we suppose that $B$ is irreducible and reduced so that $B$ is smooth by Remark 2.6. But

$$
h^{0}\left(\pi_{*} \mathcal{O}_{B}\right)=h^{0}\left(\mathcal{O}_{C}\right)+h^{0}\left(S^{k} E \otimes L\right) \geq 2
$$

cannot hold if $B$ is irreducible and reduced, hence $B$ must be neither irreducible nor reduced. So there exists an $m$-section of zero self-intersection on $X$ with $m \leq \frac{k+2}{2}$ due to Lemma 3.7, and it contradicts the minimality of $k$. Therefore, $R^{2} \neq \mathcal{O}_{C}$.

We next prove the existence of a surjection $\pi^{*} E \rightarrow R^{-1}$. Because both $\pi^{*} E$ and $R^{-1}$ have degree 0 and $\pi^{*} E$ is semi-stable, it suffices to find a nonzero morphism $\pi^{*} E \rightarrow R^{-1}$. By the adjoint property, it is equivalent to show that $\operatorname{Hom}\left(E, \pi_{*} R^{-1}\right) \neq 0$. By pushing forward the exact sequence

$$
0 \rightarrow \mathcal{O}_{X}\left(-(k+1) C_{1}-\mathfrak{b} f\right) \rightarrow \mathcal{O}_{X}\left(-C_{1}\right) \rightarrow \mathcal{O}_{D}\left(-C_{1}\right) \rightarrow 0
$$

on $X$ to $C$, we can see that

$$
\pi_{*} R^{-1} \cong R^{1} \pi_{*} \mathcal{O}_{X}\left(-(k+1) C_{1}-\mathfrak{b} f\right) \cong S^{k-1} E \otimes L^{-1} .
$$

Thus $\operatorname{Hom}\left(E, \pi_{*} R^{-1}\right) \neq 0$ follows once we prove that $\operatorname{Hom}\left(E, S^{k-1} E \otimes L^{-1}\right) \neq 0$.

For $k=3$, if $S^{3} E$ is destabilized by a line subbundle $L^{-1} \rightarrow S^{3} E$, then it induces a destabilizing subbundle $E \otimes L^{-1} \rightarrow S^{2} E$ with the quotient bundle $S^{2} E \rightarrow L^{2}$, so there is an isomorphism $E \cong E \otimes L^{2}$. Hence we have $\operatorname{Hom}\left(E, S^{2} E \otimes L^{-1}\right)=\operatorname{Hom}\left(E \otimes L^{2}, S^{2} E \otimes L^{-1}\right)=\operatorname{Hom}\left(E \otimes L^{-1}, S^{2} E\right) \neq 0$ where the second equality is obtained from twisting by $L^{-3}$ and using the fact $L^{2} \in J_{2}(C)$ (see Proposition 3.5).

For $k=4$, if $S^{4} E$ has a destabilizing subbundle $L^{-1} \rightarrow S^{4} E$, then it gives a subbundle $E \otimes L^{-1} \rightarrow S^{3} E$ which destabilizes $S^{3} E$, and we know from Proposition 3.9 that there is also a subbundle $E \otimes L \rightarrow S^{3} E$. That is, $\operatorname{Hom}\left(E, S^{3} E \otimes L^{-1}\right) \neq 0$.

For the remaining $k \geq 5$, we start with the three exact sequences

$$
\begin{gathered}
0 \rightarrow S^{k-2} E \otimes A \rightarrow E \otimes S^{k-1} E \otimes A \rightarrow S^{k} E \otimes A \rightarrow 0, \\
0 \rightarrow E \otimes S^{k-3} E \otimes A \rightarrow E \otimes E \otimes S^{k-2} E \otimes A \rightarrow S^{k-1} E \otimes A \rightarrow 0, \\
0 \rightarrow S^{k-4} E \otimes A \rightarrow E \otimes S^{k-3} E \otimes A \rightarrow S^{k-2} E \otimes A \rightarrow 0
\end{gathered}
$$

from (3.1) being twisted by $A$ and $E \otimes A$ for a line bundle $A$ on $C$. Thus we have

$$
\begin{aligned}
H^{0}\left(E \otimes S^{k-1} E \otimes A\right) \neq 0 & \Leftrightarrow H^{0}\left(S^{k} E \otimes A\right) \neq 0 \text { or } H^{0}\left(S^{k-2} E \otimes A\right) \neq 0, \\
H^{0}\left(E \otimes E \otimes S^{k-2} E \otimes A\right) \neq 0 & \Leftrightarrow H^{0}\left(E \otimes S^{k-1} E \otimes A\right) \neq 0 \text { or } H^{0}\left(E \otimes S^{k-3} E \otimes A\right) \neq 0, \\
H^{0}\left(E \otimes S^{k-3} E \otimes A\right) \neq 0 & \Leftrightarrow H^{0}\left(S^{k-2} E \otimes A\right) \neq 0 \text { or } H^{0}\left(S^{k-4} E \otimes A\right) \neq 0 .
\end{aligned}
$$


Then $H^{0}\left(S^{k} E \otimes L^{+1}\right)=\operatorname{Hom}\left(L^{-1}, S^{k} E\right) \neq 0$ implies $\operatorname{Hom}\left(E \otimes L, S^{k-1} E\right)=H^{0}\left(E \otimes S^{k-1} E \otimes L^{-1}\right) \neq 0$ from the following implication diagram whose equivalence at the middle is established by Lemma 4.12 , An arrow labeled with $\times$ indicates an implication which leads to a contradiction to the assumption that there is no $m$-section of zero self-intersection on $X$ for any $m<k$.

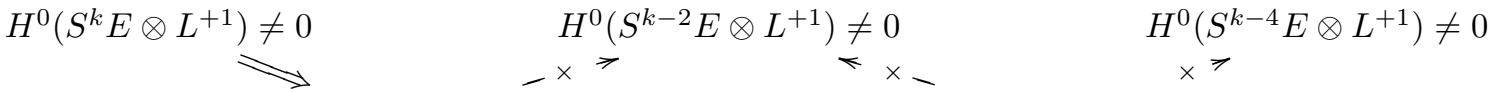

$$
\begin{aligned}
& \begin{array}{cc}
H^{0}\left(E \otimes S^{k-1} E \otimes L^{+1}\right) \neq 0 & H^{0}\left(E \otimes S^{k-3} E \otimes L^{+1}\right) \neq 0 \\
& \times>
\end{array} \\
& H^{0}\left(E \otimes E \otimes S^{k-2} E \otimes L^{+1}\right) \neq 0 \\
& \Uparrow \\
& H^{0}\left(E \otimes E \otimes S^{k-2} E \otimes L^{-1}\right) \neq 0 \\
& \Longleftarrow-x_{>} \\
& H^{0}\left(E \otimes S^{k-1} E \otimes L^{-1}\right) \neq 0 \quad H^{0}\left(E \otimes S^{k-3} E \otimes L^{-1}\right) \neq 0 \\
& \Longleftrightarrow x_{>}<x^{-} \quad-x_{>} \\
& H^{0}\left(S^{k} E \otimes L^{-1}\right) \neq 0 \quad H^{0}\left(S^{k-2} E \otimes L^{-1}\right) \neq 0 \quad H^{0}\left(S^{k-4} E \otimes L^{-1}\right) \neq 0
\end{aligned}
$$

Thus, for $k \geq 3, \pi^{*} E=R^{-1} \oplus R$ for some torsion line bundle $R$ over the unramified $k$-covering $\pi$. Finally, we complete the proof applying Lemma 4.10 .

Remark 4.14. Nori [18, p. 35] defines a vector bundle $V$ on a complete, connected, reduced scheme $X$ to be finite if there exists a finite collection $\mathcal{S}$ of vector bundles on $X$ such that for each $n \geq 0$,

$$
V^{\otimes n}=W_{1} \oplus W_{2} \oplus \cdots \oplus W_{k_{n}} \text { for some } W_{i} \in \mathcal{S} .
$$

It is also proven in the same paper that $V$ is finite if and only if $V$ is étale-trivial (over the base field of characteristic 0). Therefore, in the case where $X$ is a smooth projective curve $C$ of genus $g \geq 2$ and $V$ is a vector bundle $E$ of rank 2 with trivial determinant, Theorem 4.13 says that when $S^{2} E$ is stable, $E$ is finite if and only if $S^{k} E$ is destabilized by a line subbundle for some $k \geq 3$.

If $S^{k} E$ is not stable for some $k \geq 3$ which may assumed to be minimal, then $k=3$ or 4 or 6 due to Theorem 4.2. Moreover, in those cases, from Proposition 3.9 (with Proposition 3.1) for $k=3$, and from the proof of Theorem 4.8 for $k=4$ and 6 , we know that $S^{l} E$ is destabilized by a line subbundle for some $l \geq k$, and hence $E$ becomes finite by Theorem 4.13. As a conclusion, when $S^{2} E$ is stable, we can state that $E$ is finite if and only if $S^{k} E$ is not stable for some $k \geq 3$.

\section{REFERENCES}

[1] V. Balaji, Lectures on principal bundles, in: L. Brambila-Paz, S. B. Bradlow, O. García-Prada, S. Ramanan (Eds.), Moduli Spaces and Vector Bundles, London Math. Soc. Lecture Note Ser. 359, Cambridge Univ. Press, Cambridge, 2009. 2-28.

[2] A. Beauville, On the stability of the direct image of a generic vector bundle, Preprint (2000) available at http://math1.unice.fr/ beauvill/pubs/imdir.pdf

[3] C. Birkenhake, H. Lange, Complex Abelian Varieties. Second edition, Grundlehren der Mathematischen Wissenschaften 302, Springer-Verlag, Berlin, 2004.

[4] I. Biswas, Étale triviality of finite vector bundles over compact complex manifolds, Adv. Math. 369 (2020) 107167.

[5] I. Biswas, T. Gómez, Hecke transformation for orthogonal bundles and stability of Picard bundles, Comm. Anal. Geom. 18(5) (2010) 857-890.

[6] I. Choe, Y. Choi, S. Kim, E. Park, Bisecant and trisecant curves on ruled surfaces, J. Algebra 497 (2018) 1-18. 
[7] I. Choe, G. H. Hitching, Maximal isotropic subbundles of orthogonal bundles of odd rank over a curve, Internat. J. Math. 26(13) (2015) 1550106.

[8] Y. Choi, E. Park, On higher syzygies of ruled surfaces III, J. Pure Appl. Algebra 219(10) (2015) 4653-4666.

[9] R. Friedman, Algebraic Surfaces and Holomorphic Vector Bundles, Universitext, Springer-Verlag, New York, 1998.

[10] W. Fulton, J. Harris, Representation Theory, Graduate Texts in Mathematics 129, Springer-Verlag, New York, 1991.

[11] R. Hartshorne, Ample Subvarieties of Algebraic Varieties, Lecture Notes in Mathematics 156, Springer-Verlag, BerlinNew York, 1970

[12] R. Hartshorne, Algebraic Geometry, Graduate Texts in Mathematic 52, Springer-Verlag, New York-Heidelberg, 1977.

[13] G. H. Hitching, Subbundles of symplectic and orthogonal vector bundles over curves, Math. Nachr. 280(13-14) (2007) 1510-1517.

[14] R. Lazarsfeld, Positivity in Algebraic Geometry I 8 II, A Series of Modern Surveys in Mathematics 48 \& 49, SpringerVerlag, Berlin, 2004.

[15] M. Maruyama, Elementary transformations in the theory of algebraic vector bundles, in: J. M. Aroca, R. Buchweitz, M. Giusti, M. Merle (Eds.), Algebraic Geometry (La Rábida, 1981), Lecture Notes in Math. 961, Springer, Berlin, 1982. 241-266.

[16] D. Mumford, Theta characteristics of an algebraic curve, Ann. Sci. École Norm. Sup. (4) 4(2) (1971) $181-192$.

[17] M. S. Narasimhan, C. S. Seshadri, Stable and unitary vector bundles on a compact Riemann surface, Ann. of Math. (2) 82(3) (1965) 540-567.

[18] M. V. Nori, On the representations of the fundamental group, Compositio Math. 33(1) (1976) 29-41.

[19] S. Ramanan, Orthogonal and spin bundles over hyperelliptic curves, Proc. Indian Acad. Sci. Math. Sci. 90(2) (1981) 151-166.

[20] A. Ramanathan, Moduli for principal bundles over algebraic curves: I $\&$ II, Proc. Indian Acad. Sci. Math. Sci. 106(3) (1996) 301-328 \& 106(4) (1996) 421-449.

[21] J. Rosoff, Effective divisor classes on a ruled surface, Pacific J. Math. 202(1) (2002) 119-124.

Department of Mathematical Sciences, Kaist, 291 Daehak-ro, Yuseong-gu, Daejeon 34141, Korea

Email address: jeongseop@kaist.ac.kr 